\title{
The Relationship Between Fuzzy Soft and Soft Topologies
}

\author{
José Carlos R. Alcantud ${ }^{1}$ (D)
}

Received: 2 May 2021 /Revised: 11 September 2021/ Accepted: 17 November 2021/Published online: 7 February 2022

(C) The Author(s) 2022

\begin{abstract}
This paper attempts to forward both soft topology and fuzzy soft topology with a pioneering analysis of their mutual relationships. With each soft topology we associate a parameterized family of fuzzy soft topologies called its $t$-pushes. And each fuzzy soft topology defines a parameterized family of soft topologies called its $t$-throwbacks. Different soft topologies produce different $t$-pushes. But we prove by example that not all fuzzy soft topologies are characterized by their $t$-throwbacks. The import of these constructions is that some properties stated in one setting can be investigated in the other setting. Our conclusions should fuel future research on both fuzzy soft topology and soft topology.
\end{abstract}

Keywords Soft topology · Fuzzy soft topology ·

Topology $\cdot$ Separation axioms

\section{Introduction}

This paper lies at the crossroads of three disciplines, namely, topology, fuzzy set theory, and soft set theory.

A consensus has taken hold that topology as a welldefined mathematical discipline originates in the early 1900s, although antecedents like Euler's 1736 paper on the Seven Bridges of Königsberg can be traced back some centuries. The term "topological space" was coined by Felix Hausdorff [1] in 1914, although then both measure theory and topology were considered parts of set theory.

José Carlos R. Alcantud

jcr@usal.es

1 BORDA Research Unit and IME, University of Salamanca, Salamanca, Spain
Nowadays the Dewey Decimal Classification assigns mathematics to division 510, with subdivisions for Algebra \& Number theory, Arithmetic, Topology, Analysis, Geometry, Numerical analysis, and Probabilities \& Applied mathematics.

The Weierstrass Extreme Value Theorem shows in its modern formulation that decision-making can enjoy the benefits of topological arguments. The result joins the forces of two topological concepts (compactness of a domain of alternatives and continuity of a real-valued function defined on it). Other disciplines like mathematical economics adopted this technique, e.g., in the form of the Bergstrom-Walker theorem [2, 3] that states that any continuous acyclic relation defined on a compact topological space has a maximal element. Actually this set of sufficient conditions is also necessary [4].

It is also acknowledged that Zadeh [5] launched fuzzy set theory, and interest on fuzzy concepts mounted as new research was conducted. Topological notions were soon combined with fuzzy set theory and its extensions. Chang [6] defined fuzzy topology in 1968, and the notion was reformulated by Goguen [7] and Lowen [8]. Other models of uncertain knowledge have been combined with topological approaches too. For example, intuitionistic fuzzy topological spaces were defined in 1997 by Çoker [9].

Topology has also exerted a gravitational pull among soft set theory researchers. Soft topology was launched by Shabir and Naz [10] in 2011 and it owes to the hybridization of soft set theory [11] with the axiomatics of a topology. Many contributions showcase the properties of soft topologies, and all too often their achievements consist of a replication of pre-existent ideas from topology. Interestingly, Das and Samanta [12], Nazmul and Samanta [13], and Zorlutuna et al. [14] set forth a notion of soft point which became crucial in the analysis of properties of 
soft interior points and soft neighborhood systems. Other definitions of soft point [15] and element [16] exist. The behavior of separation axioms has been translated to a soft topological framework (Shabir and Naz [10], Al-shami and El-Shafei [17], Hussain and Ahmad [18], and Terepeta [19]). The study of soft compactness owes especially to Aygünoğlu and Aygün [15] and Zorlutuna et al. [14], and more recently, Al-shami et al. [20] have produced seven generalized classes of soft semi-compact spaces. Separability axioms are another remarkable element in the recent development of soft topology. Alcantud [21] used them to create well-behaved soft topological spaces. Kočinac et al. [22] have introduced and investigated soft Menger spaces.

Other authors fired off articles on a wide range of combinations of topology with the spirit of soft set theory. Fuzzy soft topological spaces were defined by Tanay and Kandemir [23]. They have been used as a valuable tool in multi-criteria group decision making (Khameneh et al. [24]), thus extending the applicability of fuzzy soft sets (e.g., in medical practice [25]) to a topological context. Other relevant references include Khameneh et al. [26] and Roy and Samanta [27]. Relatedly, Riaz and Tehrim [28] introduced bipolar fuzzy soft topologies, and in addition Riaz et al. [29] defined hesitant fuzzy soft topologies. Also, Riaz et al. [30] have used the extended approach by $N$-soft sets (Fatimah et al. [31]) in order to design $N$-soft topologies. Notably, both [29] and [30] give applications in decision-making.

However, researchers seem to have turned a blind eye to the relationships between soft topologies and fuzzy soft topologies to the extent that they have become two disparate fields of research. In order to fill this research gap we perform an extensive analysis of their relationships. We believe that this approach has its upside, even though the output is a mostly theoretical article. A strong theoretical foundation of this aspect of topological soft set theory should forward both disciplines and increase their appeal. Being a pioneering approach, it can also boost the analysis of relationships among other types of topological soft structures like those mentioned above.

This research paper consists of five sections. Section 2 gives preliminary notions from soft set theory, soft topology, fuzzy soft set theory, and fuzzy soft topology. We give a long list of yardstick examples of both soft and fuzzy soft topological spaces, some of which are new in this literature. In Sect. 3 we formally identify a soft topology with a fuzzy soft topology. In addition, with each soft topology we associate a parameterized family of fuzzy soft topologies called its $t$-pushes, in such way that different soft topologies produce different $t$-pushes. This process contains the aforementioned identification of a soft topology with a fuzzy soft topology as an extreme case, namely, its identification coincides with its 1-push. With this tool we lay the grounds of the investigation of which properties of a soft topology are transmitted to the fuzzy soft topologies thus defined. Section 4 studies the converse problems which we happen to find more interesting, plus the relationships among both processes. In particular, we investigate some properties of a fuzzy soft topology that pass on to the soft topologies called its $t$-throwbacks. Examples give explicit constructions that illustrate these ideas. In particular, we prove by example that different fuzzy soft topologies can produce the same collection of $t$-throwbacks. The goal of Sect. 5 is to conclude this paper with a succinct but precise recapitulation of our main findings, and to give some lines for future research. At the end of the paper we summarize notation and conventions used along the article to facilitate the reading of our results.

\section{Preliminaries}

In this paper $X$ denotes a fixed nonempty set (the universe of discourse), and $E$ denotes a set of attributes of the alternatives. For each set $U$, we denote by $\mathcal{P}(U)$ the set formed by all the subsets of $U$, i.e., the set of parts of the set $U$; and $\mathcal{F}(U)$ denotes the set formed by the fuzzy subsets of $U$, i.e., the set of mappings $U \longrightarrow[0,1]$.

In Sect. 2.1, we briefly recall the fundamental concepts of soft set theory and soft topology. Section 2.2 does the same with fuzzy soft set theory and fuzzy soft topology. The reader can consult Munkres [32] and Willard [33] for the basic notions in topology.

Sometimes we need to distinguish clearly between topologies and (fuzzy) soft topologies. In this case we use the alternative terms "crisp topology" or "point-set topology" to emphasize the classical notion of topology on a set.

\subsection{Elements of Soft Set Theory and Soft Topology}

According to [11], a soft set on $X$ is a pair $(F, E)$, where $E$ consists of all the attributes that are needed to characterize the elements of $X$, and $F$ is a mapping $F: E \longrightarrow \mathcal{P}(X)$. Thus a soft set over $X$ is a parameterized family of subsets of $X$. In mathematical terms, a soft set on $X$ is a multi-function (also called correspondence, point-to-set mapping, or multi-valued mapping) $F$ from the set of characteristics $E$ to $X$. The set of all soft sets on $X$ characterized by attributes $E$ shall be represented by $S S_{E}(X)$ or simply $S S(X)$ if $E$ is common knowledge.

For each $e \in E$, the set $F(e)$ is a subset of $X$ that is sometimes denoted as $(F, E)(e)$ for higher accuracy. It is the set of $e$-approximate elements of $X$, also called the subset of $X$ approximated by $e$. 
Suppose that $(F, E)$ is a soft set on $X$ such that $F(e)$ is finite (resp., countable) for each $e \in E$. Then we say that $(F, E)$ is a finite (resp., a countable) soft set on $X[12,13]$.

A soft set $(F, E)$ is frequently expressed as $\{(e, F(e)): e \in E\}$. It can also be represented in tabular form when the sets $X$ and $E$ are finite [34].

Two extreme examples are the null and the absolute soft sets on $X$. The null soft set $\Phi=(\Phi, E)$ satisfies $\Phi(e)=\varnothing$ for each $e \in E$ [34, Definition 2.7]. The absolute soft set $\tilde{X}=(\tilde{X}, E)$ satisfies $\tilde{X}(e)=X$ for each $e \in E[34$, Definition 2.8].

Another noteworthy example of soft set is 'soft point'. Unfortunately, some terminological confusion surrounds the use of the term 'soft point'. Let us briefly discuss this issue.

1. We shall refer to soft spots as soft sets $(F, E)$ for which there exist $x \in X$ and $e \in E$ such that $F(e)=\{x\}$ and $F\left(e^{\prime}\right)=\varnothing$ when $e^{\prime} \in E \backslash\{e\}$. We denote by $\left(\{x\}_{e}, E\right)$ this special soft set. Observe that this notion appeared in [12] under the term 'soft point', and [16, Definition 3.1] used the term 'soft element' to denote the very same concept

2. In addition, [14, Definition 3.7] defined soft points in a different way: soft points in [14] are soft sets $(F, E)$ for which there exists $e \in E$ such that $F(e) \neq \varnothing$, and $F\left(e^{\prime}\right)=\varnothing$ when $e^{\prime} \in E \backslash\{e\}$. Soft spots are a particular case of this definition.

3. However $[10,19]$ define soft points as soft sets $(F, E)$ for which there exists $x \in X$ such that $F(e)=$ $\{x\}$ for all $e \in E$; we denote by $(x, E)$ this special soft set.

4. To worsen things, [15, Definition 2.11] gives a definition of soft point that is more general than the concept in [10, 19]: soft points in [15] are soft sets $(F, E)$ for which there exists $A \subseteq E$ and $x \in X$ such that $F(e)=\{x\}$ for all $e \in A$. Soft spots satisfy this definition from [15] as well.

In this messy situation, we reserve the term 'soft point' for the concept used in $[10,19]$ that we have denoted by $(x, E)$. This is the notion that we shall need to define important axioms in soft topology (cf., Definitions 3 and 4 below).
Table 1 gives the tabular representation of five concepts defined above.

Many set-theoretic operations have been exported to soft set theory. For example, union, intersection, and inclusion are defined for $S S_{E}(X)$ as follows [34]: for any $\left(F_{1}, E\right),\left(F_{2}, E\right) \in S S_{E}(X)$,

(1) $\left(F_{1}, E\right) \sqcup\left(F_{2}, E\right)$ is the soft set $\left(F_{3}, E\right) \in S S_{E}(X)$ such that $F_{3}(e)=F_{1}(e) \cup F_{2}(e)$ for each $e \in E$. This can also be written as $\left(\left(F_{1}, E\right) \sqcup\left(F_{2}, E\right)\right)(e)=$ $F_{1}(e) \cup F_{2}(e)$ when $e \in E$.

(2) $\left(F_{1}, E\right) \sqcap\left(F_{2}, E\right)$ is the soft set $\left(F_{4}, E\right) \in S S_{E}(X)$ such that $F_{4}(e)=F_{1}(e) \cap F_{2}(e)$ for each $e \in E$. This can also be written as $\left(\left(F_{1}, E\right) \sqcap\left(F_{2}, E\right)\right)(e)=$ $F_{1}(e) \cap F_{2}(e)$ when $e \in E$.

(3) $\left(F_{1}, E\right) \sqsubseteq\left(F_{2}, E\right)$ represents the property $F_{1}(e) \subseteq$ $F_{2}(e)$ for each $e \in E$.

The complement of $(F, E) \in S S_{E}(X)$ is the soft set $(F, E)^{c}=\left(F^{c}, E\right) \in S S_{E}(X)$ such that $F^{c}(e)=X \backslash F(e)$ for each $e \in E$ [34, Definition 2.6].

Remark 1 The respective extensions of the concepts of union and intersection of two soft sets to arbitrary collections of soft sets are direct.

Also, a concept of soft equality derives from soft inclusions [34, Definition 2.4]. Thus $\left(F_{1}, E\right)=\left(F_{2}, E\right)$ means $\left(F_{1}, E\right) \sqsubseteq\left(F_{2}, E\right)$ and $\left(F_{2}, E\right) \sqsubseteq\left(F_{1}, E\right)$. In other words, $F_{1}(e)=F_{2}(e)$ for each $e \in E$.

We say that $(F, E),\left(F^{\prime}, E\right) \in S S_{E}(X)$ are disjoint when $(F, E) \sqcap\left(F^{\prime}, E\right)=\Phi$. Clearly, disjoint non-empty soft sets are different (i.e., they are not soft equal).

Example 1 For illustration, suppose $X=\left\{o_{1}, o_{2}\right\}, E=\left\{e_{1}, e_{2}, e_{3}\right\} . \quad$ Consider $\quad\left(F^{1}, E\right) \quad$ and $\left(F^{2}, E\right)$ such that:

$$
\begin{aligned}
& F^{1}\left(e_{1}\right)=\left\{o_{1}\right\}, F^{1}\left(e_{2}\right)=\varnothing, F^{1}\left(e_{3}\right)=X, \\
& F^{2}\left(e_{1}\right)=\left\{o_{1}\right\}, F^{2}\left(e_{2}\right)=X, F^{2}\left(e_{3}\right)=\left\{o_{2}\right\} .
\end{aligned}
$$

Table 2 gives the tabular representation of $\left(F^{1}, E\right)$, $\left(F^{2}, E\right),\left(F_{1}, E\right) \sqcup\left(F_{2}, E\right),\left(F_{1}, E\right) \sqcap\left(F_{2}, E\right)$ and $\left(F_{1}^{c}, E\right)$.

We are in a position to define soft topology on $X$ (with a fixed set of attributes $E$ ). It consists of a family $\tau \subseteq S S_{E}(X)$ that satisfies the following definition:

Table 1 The tabular representation of the null soft set, a soft spot $\left(\left\{o_{1}\right\}_{e}, E\right)$, a soft point $\left(o_{1}, E\right)$, a soft point $(F, E)$ in the sense of [14], and the full soft set $\left(X=\left\{o_{1}, o_{2}\right\}, E=\left\{e, e^{\prime}\right\}\right)$

\begin{tabular}{lllllllllllllll}
\hline$(\Phi, \mathrm{E})$ & $\mathrm{e}$ & $\mathrm{e}^{\prime}$ & $\left(\left\{o_{1}\right\}_{e}, \mathrm{E}\right)$ & $\mathrm{e}$ & $\mathrm{e}^{\prime}$ & $\left(o_{1}, \mathrm{E}\right)$ & $\mathrm{e}$ & $\mathrm{e}^{\prime}$ & $(\mathrm{F}, \mathrm{E})$ & $\mathrm{e}$ & $\mathrm{e}^{\prime}$ & $(\tilde{X}, \mathrm{E})$ & $\mathrm{e}$ & $\mathrm{e}^{\prime}$ \\
\hline$o_{1}$ & 0 & 0 & $o_{1}$ & 1 & 0 & $o_{1}$ & 1 & 1 & $o_{1}$ & 1 & 0 & $o_{1}$ & 1 & 1 \\
$o_{2}$ & 0 & 0 & $o_{2}$ & 0 & 0 & $o_{2}$ & 0 & 0 & $o_{2}$ & 1 & 0 & $o_{2}$ & 1 & 1 \\
\hline
\end{tabular}


Table 2 The tabular representation of the concepts of soft union, intersection and complement in Example 1

\begin{tabular}{llllllllllllllllllll}
\hline$\left(F^{1}, \mathrm{E}\right)$ & $e_{1}$ & $e_{2}$ & $e_{3}$ & $\left(F^{2}, \mathrm{E}\right)$ & $e_{1}$ & $e_{2}$ & $e_{3}$ & $\left(F^{1} \sqcup F_{2}, \mathrm{E}\right)$ & $e_{1}$ & $e_{2}$ & $e_{3}$ & $\left(F^{1} \sqcap F_{2}, \mathrm{E}\right)$ & $e_{1}$ & $e_{2}$ & $e_{3}$ & $\left(\left(F^{1}\right)^{c}, \mathrm{E}\right)$ & $e_{1}$ & $e_{2}$ & $e_{3}$ \\
\hline$o_{1}$ & 1 & 0 & 1 & $o_{1}$ & 1 & 1 & 0 & $o_{1}$ & 1 & 1 & 1 & $o_{1}$ & & 1 & 0 & 0 & $o_{1}$ & \\
$o_{2}$ & 0 & 0 & 1 & $o_{2}$ & 0 & 1 & 1 & $o_{2}$ & 0 & 1 & 1 & $o_{2}$ & & 0 & 0 & 1 & $o_{2}$ & 1 & 0 \\
\hline
\end{tabular}

Definition 1 [10, 35] A soft topology $\tau$ on $X$ is $\tau \subseteq S S_{E}(X)$, a collection of soft sets on $X$, that satisfies:

(1) $\Phi, \tilde{X} \in \tau$.

(2) The union of soft sets in $\tau$ belongs to $\tau$.

(3) The intersection of a finite number of soft sets in $\tau$ belongs to $\tau$.

The members of $\tau$ are called soft open sets or $\tau$-soft open sets for better clarity.

Example 2 Here is a brief list of examples of soft topologies on a set $X$, some of which are new in the literature:

(i) The indiscrete soft topology is $\tau_{i d}=\{\Phi, \tilde{X}\}$, and the discrete soft topology is $\tau_{d}=S S_{E}(X)$ [10].

(ii) Suppose $(F, E) \in S S_{E}(X)$. Then $\tau[F]=$ $\{\Phi, \tilde{X},(F, E)\}$ is a soft topology. It coincides with the indiscrete soft topology when either $(F, E)=\Phi$ or $(F, E)=\tilde{X}$.

(iii) Suppose $(F, E) \in S S_{E}(X)$. Then $\tau[F+]=$ $\left\{\Phi, \tilde{X},(F, E),\left(F^{c}, E\right)\right\}$ is a soft topology because $\quad \Phi=(F, E) \sqcap\left(F^{c}, E\right) \quad$ and $\tilde{X}=(F, E) \sqcup\left(F^{c}, E\right)$. It coincides with the indiscrete soft topology when either $(F, E)=$ $\Phi$ or $(F, E)=\tilde{X}$.

(iv) The included point-to-attribute soft topologies are defined as follows: if we fix $x \in X$ and $e \in E$,

$\tau_{(x, e)}=\left\{(F, E) \in S S_{E}(X)\right.$ such that

$x \in F(e)\} \cup\{\Phi\}$

(v) The excluded point-to-attribute soft topologies are defined as follows: if we fix $x \in X$ and $e \in E$,

$\tau_{(\bar{x}, e)}=\left\{(F, E) \in S S_{E}(X)\right.$ such that

$x \notin F(e)\} \cup\{\tilde{X}\}$

(vi) The included point soft topologies are defined as follows: if we fix $x \in X$,
$\tau_{(x)}=\left\{(F, E) \in S S_{E}(X)\right.$ such that $x \in F(e)$

for each $e \in E\} \cup\{\Phi\}$

(vii) The excluded point soft topologies are defined as follows: if we fix $x \in X$,

$\tau_{(\bar{x})}=\left\{(F, E) \in S S_{E}(X)\right.$ such that $x \notin F(e)$

for each $e \in E\} \cup\{\tilde{X}\}$

(viii) The cofinite soft topology [13] is defined as $\tau_{c}=\left\{(F, E) \in S S_{E}(X)\right.$ such that $(F, E)^{c}$ is

a finite soft set $\}$

When $\tau, \tau^{\prime} \subseteq S S_{E}(X)$ are soft topologies, $\tau^{\prime}$ is finer than $\tau$ (or $\tau$ is coarser than $\tau^{\prime}$ ) when $\tau \subseteq \tau^{\prime}$.

Example 3 Let us now establish some comparisons among soft topologies:

1. The coarsest soft topology is the indiscrete soft topology $\tau_{i d}=\{\Phi, \tilde{X}\}$, and the finest soft topology is the discrete soft topology $\tau_{d}=S S_{E}(X)$ [10].

2. For each $x \in X$ and $e \in E, \tau_{(x, e)}$ is finer than $\tau_{(x)}$ because $(F, E) \in \tau_{(x)}$ implies $(F, E) \in \tau_{(x, e)}$.

3. Likewise, for each $x \in X$ and $e \in E, \tau_{(\bar{x}, e)}$ is finer than $\tau_{(\bar{x})}$ because $(F, E) \in \tau_{(\bar{x})}$ implies $(F, E) \in \tau_{(\bar{x}, e)}$.

4. When $(F, E) \in S S_{E}(X)$ and neither $(F, E)=\Phi$ nor $(F, E)=\tilde{X}$ are true, there exists $x \in F(e)$ for some $x \in$ $X$ and $e \in E$. Then $\tau[F]$ is coarser than $\tau_{(x, e)}$.

5. When $(F, E) \in S S_{E}(X)$ and $x \notin F(e)$ for some $x \in X$ and $e \in E, \tau[F]$ is also coarser than $\tau_{(\bar{x}, e)}$.

Example 4 When $\left(F_{1}, E\right), \ldots,\left(F_{r}, E\right) \in S S_{E}(X)$ are such that $\left(F_{1}, E\right) \sqsubseteq\left(F_{2}, E\right) \sqsubseteq \cdots \sqsubseteq\left(F_{r}, E\right), \quad$ the family $\left\{\Phi, \tilde{X},\left(F_{1}, E\right), \ldots,\left(F_{r}, E\right)\right\}$ is a soft topology.

Furthermore, general procedures exist that produce soft topologies from point-set topologies. First we recall a very direct construction: 
Definition $2[19,21]$ Suppose that $\Sigma=\left\{\Sigma_{e}\right\}_{e \in E}$ is a family of point-set topologies on $X$. Then

$\tau(\Sigma)=\left\{\{(e, F(e)): e \in E\} \in S S_{E}(X)\right.$ such that $F(e) \in \Sigma_{e}$ for each $e \in E\}$

defines a soft topology on $X$. It is called the soft topology on $X$ generated by $\Sigma$.

We can also write $\tau(\boldsymbol{\Sigma})=\tau(\Sigma)$ if $\Sigma_{e}=\Sigma_{e^{\prime}}=\Sigma$ for each $e, e^{\prime} \in E$.

Alcantud [21] applies the construction above to prove that $\tau_{c}=\tau\left(\Sigma_{c}\right)$, i.e., the cofinite soft topology can be obtained from the cofinite crisp topology on $X$, by the process in Definition 2.

The second procedure that generates soft topologies from crisp topologies relies on the behavior of soft open bases. Let us summarize some preliminary facts.

Quite naturally, soft bases are defined as collections of soft sets that generate soft topologies by taking the set formed by the arbitrary soft unions of their elements. So $\mathcal{B} \subseteq \tau$ is a soft base for the soft topology $\tau$ when every $(F, E) \in \tau$ can be expressed as a union of soft sets from $\mathcal{B}$ [35]. Roy and Samanta produced the more operative concept of a soft open base. Any soft open base can be used to generate a soft topology [36, Theorem 13] in such way that the soft open base is a soft base of the topology that it generates by this process [36, Theorem 16].

Alcantud [21, Proposition 1] proves that any base for a crisp topology produces a soft open base by an explicit definition. Now a soft topology ensues from this soft open base [36, Theorem 13]. Thus any base for a crisp topology defines an associated soft topology.

Notice that the two constructions of soft topologies defined above are closely related. Suppose that we fix a base for a crisp topology. We can produce both a crisp topology and a soft open base from it. Then we can apply Definition 2 to this crisp topology, and we can also produce a soft topology from the soft open base. The conclusion is that the respective soft topologies that we obtain are the same [21, Theorem 3].

Section 1 explains that the literature has provided many axioms like soft compactness, soft connectedness, soft separability, or soft separation axioms. Here we shall only refer to some soft separation axioms.

Definition 3 [10, 19] A soft topology $\tau$ on $X$ is $T_{0}$ when for all $x, y \in X, x \neq y$, either there is $(F, E) \in \tau$ with $(x, E) \sqsubseteq(F, E)$ such that $(y, E) \sqsubseteq(F, E)$ is false, or there is $(G, E) \in \tau$ with $(y, E) \sqsubseteq(G, E)$ such that $(x, E) \sqsubseteq(G, E)$ is false.
Notice that $(x, E) \sqsubseteq(F, E)$ is equivalent to $x \in F(e)$ for each $e \in E$. It is sometimes convenient to abbreviate $(x, E) \sqsubseteq(F, E)$ by the shorthand $x \in(F, E)$, as done in [10, 19]. Then axiom $T_{0}$ adopts the following form: for all $x, y \in X, x \neq y$, either there is $(F, E) \in \tau$ with $x \in(F, E)$ and $y \notin(F, E)$,or there is $(G, E) \in \tau$ with $y \in(G, E)$ and $x \notin(G, E)$. Likewise one can define:

Definition 4 [10, 19] A soft topology $\tau$ on $X$ is $T_{1}$ when for all $x, y \in X, x \neq y$, there is $(F, E) \in \tau$ with $x \in(F, E)$ and $y \notin(F, E)$, and there is $(G, E) \in \tau$ with $y \in(G, E)$ and $x \notin(G, E)$.

And it is $T_{2}$ when for all $x, y \in X, x \neq y$, there are disjoint $(F, E),(G, E) \in \tau$ with $x \in(F, E)$ and $y \in(G, E)$.

Terepeta [19, Theorem 4] proves that if $\Sigma$ is a $T_{0}$, resp., $T_{1}, T_{2}$, point-set topology on $X$, then $\tau(\Sigma)$ is $T_{0}$, resp., $T_{1}$, $T_{2}$. This is also true for other separation axioms like $T_{3}$ and $T_{4}$ that we do not refer to in this paper.

\subsection{Elements of Fuzzy Soft set Theory and Fuzzy Soft Topology}

According to [37], a fuzzy soft set on $X$ is a pair $(f, E)$, where $E$ consists of all the attributes that are needed to characterize the elements of $X$, and $f$ is a mapping $f: E \longrightarrow \mathcal{F}(X)$. The set of all fuzzy soft sets on $X$ with attributes $E$ will be denoted by $F S S_{E}(X)$ or simply $\operatorname{FSS}(X)$ if $E$ is common knowledge.

A fuzzy soft set on $X$ associates with each $e \in E$, the fuzzy subset $f(e)$ of $X$ consisting of the members that (partially) satisfy $e$. By convenience, the notation $f_{e}(x)$ is frequently adopted to denote $f(e)(x)$, the degree of membership of $x$ to the fuzzy subset of $E$ defined by $e$. For higher accuracy we sometimes denote the fuzzy subset $f(e)$ of $X$ by $(f, E)(e)$. As in the case of soft set, fuzzy soft sets can also be represented in tabular form when both $X$ and $E$ are finite.

The null and the absolute fuzzy soft sets on $X$ are respectively denoted by $\hat{\Phi}=(\hat{\Phi}, E)$ and $\hat{X}=(\hat{X}, E)$. The null fuzzy soft set $\hat{\Phi}$ is characterized by $\hat{\Phi}(e)(x)=0$ for each $e \in E$ and $x \in X$, whereas the absolute fuzzy soft set $\hat{X}$ satisfies $\hat{X}(e)(x)=1$ for each $e \in E$ and $x \in X$ [37].

Union, intersection, and inclusion are defined for $F_{S S}(X)$ as follows [27, 37]: for any $\left(f_{1}, E\right),\left(f_{2}, E\right) \in F S S_{E}(X)$,

(1) $\left(f_{1}, E\right) \cup \hat{\cup}\left(f_{2}, E\right)$ is the fuzzy soft set $\left(f_{3}, E\right) \in F S S_{E}(X)$ such that $f_{3}(e)(x)=\max \left\{f_{1}(e)(x), f_{2}(e)(x)\right\}$ for each $e \in E$ and $x \in X$.

(2) $\quad\left(f_{1}, E\right) \hat{\sqcap}\left(f_{2}, E\right)$ is the fuzzy soft set $\left(f_{4}, E\right) \in F_{S S}(X)$ such that $f_{4}(e)(x)=\min \left\{f_{1}(e)(x), f_{2}(e)(x)\right\}$ for each $e \in E$ and $x \in X$. 
(3) $\left(f_{1}, E\right) \hat{\sqsubseteq}\left(f_{2}, E\right)$ represents the property $f_{1}(e)(x) \leqslant$ $f_{2}(e)(x)$ for each $e \in E$ and $x \in X$.

Remark 2 The respective extensions of the concepts of union and intersection of two fuzzy soft sets to arbitrary collections of fuzzy soft sets are direct.

Example 5 Let us fix $X=\left\{o_{1}, o_{2}\right\}$ and $E=\left\{e_{1}, e_{2}\right\}$. Consider $\left(f^{1}, E\right),\left(f^{2}, E\right)$, and $\left(f^{3}, E\right)$, three fuzzy soft sets whose tabular representations are given in Table 3 .

Then Table 4 gives the tabular representation of the fuzzy soft union and intersection of $\left(f^{1}, E\right)$ and $\left(f^{2}, E\right)$. Now we can observe that $\left(f_{3}, E\right) \hat{\sqsubseteq}\left(f_{2}, E\right)$ is false, and $\left(f_{3}, E\right) \hat{\complement}\left(f^{1}, E\right) \hat{\sqcup}\left(f^{2}, E\right)$ holds true.

A special type of fuzzy soft set is formed by fuzzy soft points [26, Definition 6.2]. Let us fix $x \in X$ and $t \in(0,1]$. Then the fuzzy soft point $\left(\{x\}_{t}, E\right)$ is the fuzzy soft set for which

$$
\begin{aligned}
\{x\}_{t}: E & \longrightarrow \mathcal{F}(X) \\
e & \mapsto\{x\}_{t}(e) \text { suchthat }\{\mathrm{x}\}_{\mathrm{t}}(\mathrm{e})(\mathrm{z})=\left\{\begin{array}{c}
\mathrm{t} \text { whenz }=\mathrm{x}, \\
0 \text { otherwise }
\end{array}\right.
\end{aligned}
$$

For illustration, notice that the fuzzy soft set $\left(f^{1}, E\right)$ in Example 5 is the fuzzy soft point $\left(\left\{o_{1}\right\}_{0.7}, E\right)$.

Remark 3 The use of the term 'fuzzy soft point' is not free from difficulties either. We recommend [26, Section 6] for a discussion of earlier definitions.

The fuzzy soft points $\left(\{x\}_{t}, E\right)$ and $\left(\{y\}_{t^{\prime}}, E\right)$ are distinct when $x \neq y$ [26, Definition 6.2]. This is equivalent to claiming $\left(\{x\}_{t}, E\right) \hat{\sqcap}\left(\{y\}_{t^{\prime}}, E\right)=\hat{\Phi}$.

Notice that $\left(\{x\}_{t}, E\right) \hat{\sqsubseteq}(f, E)$ is equivalent to $f(e)(x) \geqslant t$ for each $e \in E$.

We say that $(f, E),\left(f^{\prime}, E\right) \in F S S_{E}(X)$ are disjoint when $(f, E) \hat{\sqcap}\left(f^{\prime}, E\right)=\hat{\Phi}$ [37]. Clearly, disjoint non-empty fuzzy soft sets are different.

With these ideas in mind, we are ready to define fuzzy soft topology on $X$ (the set of attributes being $E$ ). It is formed by a family $\hat{\tau} \subseteq F S S_{E}(X)$ that satisfies the properties in our next definition:

Table 3 The tabular representation of the fuzzy soft sets in Example 5

\begin{tabular}{lllllllll}
\hline$\left(f^{1}, \mathrm{E}\right)$ & $e_{1}$ & $e_{2}$ & $\left(f^{2}, \mathrm{E}\right)$ & $e_{1}$ & $e_{2}$ & $\left(f^{3}, \mathrm{E}\right)$ & $e_{1}$ & $e_{2}$ \\
\hline$o_{1}$ & 0.7 & 0.7 & $o_{1}$ & 0.8 & 0.5 & $o_{1}$ & 0.8 & 0.6 \\
$o_{2}$ & 0 & 0 & $o_{2}$ & 0.5 & 0.1 & $o_{2}$ & 0.2 & 0 \\
\hline
\end{tabular}

Table 4 The tabular representation of the concepts of fuzzy soft union and intersection in Example 5

\begin{tabular}{llllll}
\hline$\left(f^{1}, \mathrm{E}\right) \sqcup \hat{\imath}\left(f^{2}, \mathrm{E}\right)$ & $e_{1}$ & $e_{2}$ & $\left(f^{1}, \mathrm{E}\right) \hat{\sqcap}\left(f^{2}, \mathrm{E}\right)$ & $e_{1}$ & $e_{2}$ \\
\hline$o_{1}$ & 0.8 & 0.7 & $o_{1}$ & 0.7 & 0.5 \\
$o_{2}$ & 0.5 & 0.1 & $o_{2}$ & 0 & 0 \\
\hline
\end{tabular}

Definition 5 [27] A fuzzy soft topology $\hat{\tau}$ on $X$ is $\hat{\tau} \subseteq F_{S S}(X)$, a family of fuzzy soft sets on $X$ such that:

(1) $\hat{\Phi}, \hat{X} \in \hat{\tau}$.

(2) The union of fuzzy soft sets in $\hat{\tau}$ belongs to $\hat{\tau}$.

(3) The intersection of a finite number of fuzzy soft sets in $\hat{\tau}$ belongs to $\hat{\tau}$.

The members of $\hat{\tau}$ are called fuzzy soft open sets or $\hat{\tau}$-fuzzy soft open sets for better clarity.

Prior to this definition, a slightly different notion of fuzzy soft topology had been proposed [23]. Anyhow, both approaches owe to the procedure for defining fuzzy topologies given in [6]. Here we adopt the notion of fuzzy soft topology in the sense of [27] that satisfies the adapted version of the De Morgan's laws.

As is natural, when $\hat{\tau}, \hat{\tau}^{\prime} \subseteq F S S_{E}(X)$ are fuzzy soft topologies, we say that $\hat{\tau}$ is coarser than $\hat{\tau}^{\prime}$ (or $\hat{\tau}^{\prime}$ is finer than $\hat{\tau}$ ) when $\hat{\tau} \subseteq \hat{\tau}^{\prime}$.

For each $(f, E) \in F S S_{E}(X)$ we denote

$(f, E)^{\uparrow}=\left\{\left(f^{\prime}, E\right) \in F_{S S}(X) \mid f^{\prime}(e)(x) \geqslant f(e)(x), \forall e \in\right.$

$E, \forall x \in X\}$

and

$$
\begin{aligned}
& (f, E)^{\downarrow}=\left\{\left(f^{\prime}, E\right) \in F S S_{E}(X) \mid f^{\prime}(e)(x) \leqslant f(e)(x), \forall e \in\right. \\
& E, \forall x \in X\} .
\end{aligned}
$$

Notice that $\hat{X} \in(f, E)^{\uparrow}$ and $\hat{\Phi} \in(f, E)^{\downarrow}$.

Example 6 Here is a brief list of examples of fuzzy soft topologies on a set $X$, some of which are new in the literature:

(i) The indiscrete fuzzy soft topology is $\hat{\tau}_{i d}=\{\hat{\Phi}, \hat{X}\}$, and the discrete fuzzy soft topology is $\hat{\tau}_{d}=F S S_{E}(X) \quad$ [26, Example 4.2]. They are respectively the coarsest and finest fuzzy soft topologies on $X$.

(ii) For each $t \in[0,1]$ : 
$\hat{\tau}\left[t^{\uparrow}\right]=\left\{(f, E) \in F_{S S S_{E}}(X) \mid f(e)(x) \geqslant t, \forall e \in\right.$

$E, \forall x \in X\} \cup\{\hat{\Phi}\}$

and

$\hat{\tau}\left[t^{\downarrow}\right]=\left\{(f, E) \in F S S_{E}(X) \mid f(e)(x) \leqslant t, \forall e \in\right.$

$E, \forall x \in X\} \cup\{\hat{X}\}$

are respectively called the $t$-upper and $t$-lower fuzzy soft topologies on $X$.

(iii)

For each $(f, E) \in F S S_{E}(X)$ :

$$
\begin{aligned}
\hat{\tau}\left[f^{\uparrow}\right] & =(f, E)^{\uparrow} \cup\{\hat{\Phi}\} \\
= & \left\{\left(f^{\prime}, E\right) \in F S S_{E}(X) \mid f^{\prime}(e)(x) \geqslant f(e)(x),\right. \\
& \forall e \in E, \forall x \in X\} \cup\{\hat{\Phi}\}
\end{aligned}
$$

defines the upper contour fuzzy soft topology derived from $(f, E)$.

(iv) For each $(f, E) \in F S S_{E}(X)$ :

$$
\begin{aligned}
\hat{\imath}\left[f^{\downarrow}\right] & =(f, E)^{\downarrow} \cup\{\hat{X}\} \\
= & \left\{\left(f^{\prime}, E\right) \in F S_{E}(X) \mid f^{\prime}(e)(x) \leqslant f(e)(x),\right. \\
& \forall e \in E, \forall x \in X\} \cup\{\hat{X}\}
\end{aligned}
$$

defines the lower contour fuzzy soft topology derived from $(f, E)$.

(v) For any $t \in[0,1]$, the $t$-included point-toattribute fuzzy soft topologies are defined as follows: if we fix $x \in X$ and $e \in E$,

$$
\begin{aligned}
& \hat{\tau}_{(x, e)}^{t}=\left\{(f, E) \in F_{S S}(X)\right. \\
& \text { such that } f(e)(x) \geqslant t\} \cup\{\hat{\Phi}\}
\end{aligned}
$$

(vi) For any $t \in[0,1]$, the $t$-excluded point-toattribute fuzzy soft topologies are defined as follows: if we fix $x \in X$ and $e \in E$,

$$
\begin{aligned}
& \hat{\tau}_{(\bar{x}, e)}^{t}=\left\{(f, E) \in F S_{E}(X)\right. \\
& \text { such that } f(e)(x) \leqslant t\} \cup\{\hat{X}\}
\end{aligned}
$$

(vii) For any $t \in[0,1]$, the $t$-included point fuzzy soft topologies are defined as follows: if we fix $x \in X$ and $e \in E$,

$$
\begin{aligned}
& \hat{\tau}_{(x)}^{t}=\left\{(f, E) \in F S S_{E}(X) \text { such that } f(e)(x) \geqslant t\right. \\
& \text { for each } e \in E\} \cup\{\hat{\Phi}\}
\end{aligned}
$$

(viii) For any $t \in[0,1]$, the $t$-excluded point fuzzy soft topologies are defined as follows: if we fix $x \in X$ and $e \in E$,

$$
\begin{aligned}
& \hat{\tau}_{(\bar{x})}^{t}=\left\{(f, E) \in F S S_{E}(X) \text { such that } f(e)(x) \leqslant t\right. \\
& \text { for each } e \in E\} \cup\{\hat{X}\}
\end{aligned}
$$

Example 7 Let us now establish some comparisons among fuzzy soft topologies:

1. Let us fix $t \in[0,1]$. Then it must be the case that for each $x \in X, \hat{\tau}_{(x)}^{t}$ is finer than $\hat{\tau}\left[t^{\dagger}\right]$ because $(f, E) \in \hat{\tau}\left[t^{\dagger}\right]$ implies $(f, E) \in \hat{\tau}_{(x)}^{t}$. Besides, if we fix $t \in[0,1]$, then for each $x \in X$ and $e \in E, \hat{\tau}_{(x, e)}^{t}$ is finer than $\hat{\tau}_{(x)}^{t}$ because $(f, E) \in \hat{\tau}_{(x)}^{t}$ implies $(f, E) \in \hat{\tau}_{(x, e)}^{t}$.

2. When $(f, E) \in F S S_{E}(X)$ is such that $f(e)(x) \geqslant t$ for some $x \in X$ and $e \in E$, then $\hat{\tau}_{(x, e)}^{t}$ is also finer than $\hat{\tau}\left[f^{\dagger}\right]$. If in fact $(f, E) \in F S S_{E}(X)$ is such that for some $x \in X, f(e)(x) \geqslant t$ for each $e \in E$, then $\hat{\tau}_{(x)}^{t}$ is also finer than $\hat{\tau}\left[f^{\uparrow}\right]$.

3. When $(f, E) \in F S S_{E}(X)$ is such that $f(e)(x) \geqslant t$ for all $x \in X$ and $e \in E$, then $\hat{\tau}\left[t^{\uparrow}\right]$ is also finer than $\hat{\tau}\left[f^{\uparrow}\right]$.

4. Let us fix $t \in[0,1]$. Then it must be the case that for each $x \in X, \hat{\tau}_{(\bar{x})}^{t}$ is finer than $\hat{\tau}\left[t^{\downarrow}\right]$ because $(f, E) \in \hat{\tau}[t]$ implies $(f, E) \in \hat{\tau}_{(\bar{x})}^{t}$. Besides, if we fix $t \in[0,1]$, then for each $x \in X$ and $e \in E, \hat{\tau}_{(\bar{x}, e)}^{t}$ is finer than $\hat{\tau}_{(\bar{x})}^{t}$ because $(f, E) \in \hat{\tau}_{(\bar{x})}^{t}$ implies $(f, E) \in \hat{\tau}_{(\bar{x}, e)}^{t}$.

5. When $(f, E) \in F S_{E}(X)$ is such that $f(e)(x) \leqslant t$ for some $x \in X$ and $e \in E$, then $\hat{\tau}_{(\bar{x}, e)}^{t}$ is also finer than $\hat{\tau}\left[f^{\downarrow}\right]$. If in fact $(f, E) \in F S S_{E}(X)$ is such that for some $x \in X, f(e)(x) \leqslant t$ for each $e \in E$, then $\hat{\tau}_{(\bar{x})}^{t}$ is also finer than $\hat{\tau}\left[f^{\downarrow}\right]$.

6. When $(f, E) \in F S S_{E}(X)$ is such that $f(e)(x) \leqslant t$ for all $x \in X$ and $e \in E$, then $\hat{\tau}\left[t^{\downarrow}\right]$ is also finer than $\hat{\tau}\left[f^{\downarrow}\right]$.

Example 8 When $\left(f^{1}, E\right), \ldots,\left(f^{r}, E\right) \in F S S_{E}(X)$ are such that $\left(f^{1}, E\right) \hat{\sqsubseteq}\left(f^{2}, E\right) \hat{\sqsubseteq} \ldots \hat{\complement}\left(f^{r}, E\right)$, the family

$\left\{\hat{\Phi}, \hat{X},\left(f^{1}, E\right), \ldots,\left(f^{r}, E\right)\right\}$ is a fuzzy soft topology.

Let us now recall some definitions of fuzzy soft separation axioms. One should bear in mind that the property $\left(\{x\}_{t}, E\right) \hat{\complement}(f, E)$ is equivalent to $f(e)(x) \geqslant t$ for each $e \in E$.

Definition 6 [26] A fuzzy soft topology $\hat{\tau}$ on $X$ is: 
(i) $T_{0}$ when for all $x, y \in X, x \neq y$, and all $t, t^{\prime} \in(0,1]$, either there is $(f, E) \in \hat{\tau}$ with $\left(\{x\}_{t}, E\right) \hat{\sqsubseteq}(f, E)$ such that $\left(\{y\}_{t^{\prime}}, E\right) \hat{\sqsubseteq}(f, E)$ is false, or there is $(g, E) \in \hat{\tau}$ with $\left(\{y\}_{t^{\prime}}, E\right) \hat{\sqsubseteq}(g, E)$ such that $\left(\{x\}_{t}, E\right) \hat{\sqsubseteq}(g, E)$ is false.

(ii) $T_{1}$ when for all $x, y \in X, x \neq y$, and all $t, t^{\prime} \in(0,1]$, either there is $(f, E) \in \hat{\tau}$ with $\left(\{x\}_{t}, E\right) \hat{\sqsubseteq}(f, E)$ such that $\left(\{y\}_{t^{\prime}}, E\right) \hat{\sqsubseteq}(f, E)$ is false, and there is $(g, E) \in \hat{\tau}$ with $\left(\{y\}_{t^{\prime}}, E\right) \hat{\sqsubseteq}(g, E)$ such that $\left(\{x\}_{t}, E\right) \hat{\sqsubseteq}(g, E)$ is false.

(iii) $T_{2}$ when for all $x, y \in X, x \neq y$, and all $t, t^{\prime} \in(0,1]$, there are disjoint $(f, E),(g, E) \in \hat{\tau}$ with $\left(\{x\}_{t}, E\right) \hat{\sqsubseteq}(f, E)$ and $\left(\{y\}_{t^{\prime}}, E\right) \hat{\underline{E}}(g, E)$.

Fuzzy soft bases are collections of fuzzy soft sets that generate fuzzy soft topologies by taking the set formed by their arbitrary fuzzy soft unions. So $\mathcal{B} \subseteq \hat{\tau}$ is a fuzzy soft base for the fuzzy soft topology $\hat{\tau}$ when every $(f, E) \in \hat{\tau}$ can be expressed as a union of fuzzy soft sets from $\mathcal{B}[27$, Definition 3.9 and Theorem 3.12]. See also [26, Theorem 4.9] for a characterization of which families of fuzzy soft sets behave as a fuzzy soft base for a fuzzy soft topology.

From each fuzzy soft topology on a set $X$, some related fuzzy soft topologies on $X$ can be defined:

Proposition 1 Suppose that $\hat{\tau}$ is a fuzzy soft topology on $X$. Then

1. The set $\hat{\tau}^{\uparrow}=\bigcup\left\{(f, E)^{\uparrow} \mid(f, E) \in \hat{\tau}\right\} \cup\{\hat{\Phi}\}$ defines $a$ fuzzy soft topology on X. We call it the upper interval fuzzy soft topology derived from $\hat{\tau}$.

2. The set $\hat{\tau}^{\downarrow}=\bigcup\left\{(f, E)^{\downarrow} \mid(f, E) \in \hat{\tau}\right\} \cup\{\hat{X}\}$ defines $a$ fuzzy soft topology on $X$. We call it the lower interval fuzzy soft topology derived from $\hat{\tau}$.

The proof of Proposition 1 is routine.

\section{Fuzzy Soft Topologies Generated by Soft Topological Spaces}

This section is dedicated to the generation of fuzzy soft topologies on a set from a given soft topology on the same set. First we consider a predictable fact: the existence of a natural embedding that allows one to regard any soft topological space as a fuzzy soft topological space. Despite its reasonability we are not aware of any formal proof of this remarkable immersion. Then we produce a more general construction that embeds the previous one as a particular case of a collection of fuzzy soft topological spaces derived from any soft topological space. Finally in this section, we demonstrate that some properties of a soft topological space can be transferred to the fuzzy soft topological spaces constructed in that way.

\subsection{Soft Topologies 'are' Fuzzy Soft Topologies}

We proceed to show how one can design a natural identification of soft topologies with fuzzy soft topologies. As explained above this result will be superseded by the more general approach taken in Sect. 3.2. However the embedding is important in its own right and we believe that it deserves an explicit presentation and terminology.

The constructions in this section and also in Sect. 4 rely on the following 'folk' theorem:

Theorem 1 There is an embedding of $S S_{E}(X)$ within $\operatorname{FSS}_{E}(X)$.

The statement claims that there is an embedding. By this we mean a mapping $\eta: S S_{E}(X) \longrightarrow F S S_{E}(X)$ that is injective and preserves unions and intersections.

Proof of Theorem 1 To define one such embedding $\eta$, we recall that for each $A \in \mathcal{P}(X)$, the characteristic function of $A$ is $\chi_{A}: X \longrightarrow\{0,1\}$ such that $\chi_{A}(x)=1$ if $x \in A$, and $\chi_{A}(x)=0$ if $x \notin A$. With its aid we can identify subsets of $X$ or members of $\mathcal{P}(X)$, with fuzzy subsets of $X$, since we can also identify $\chi_{A}$ with the fuzzy subset $\chi_{A}: X \longrightarrow[0,1]$.

Define the mapping $\eta$ as follows: for each $(F, E) \in S S_{E}(X), \eta(F, E)=\left(f_{F}, E\right) \in F S S_{E}(X)$ is such that $f_{F}: E \longrightarrow \mathcal{F}(X)$

$$
\text { e } f_{F}(e)=\chi_{F(e)}
$$

It is now simple to check that $\eta$ is injective, and also that it satisfies the following properties:

1. $\eta(\Phi)=\hat{\Phi}$ and $\eta(\tilde{X})=\hat{X}$,

2. $\eta\left(\Pi_{i \in I}\left(F_{i}, E\right)\right)=\hat{\Pi_{i \in I}}\left(\eta\left(F_{i}, E\right)\right)$, and

3. $\eta\left(\sqcup_{i \in I}\left(F_{i}, E\right)\right)=\hat{\sqcup}_{i \in I}\left(\eta\left(F_{i}, E\right)\right)$.

We are ready to define an identification of soft topologies with fuzzy soft topologies on the same universe:

Definition 7 The soft topology $\tau$ on $X$ defines $\hat{\tau}^{\eta}=\{\eta(F, E) \mid(F, E) \in \tau\} \subseteq F S S_{E}(X)$. We say that $\hat{\tau}^{\eta}$ is the fuzzy soft topology associated with $\tau$.

The claim in this definition leans on the following fact whose proof is routine:

Proposition 2 Suppose that $\tau$ is a soft topology on $X$. Then $\hat{\tau}^{\eta}$ is a fuzzy soft topology on $X$.

Proof Theorem 1 guarantees the requirements for a fuzzy soft topology. 
Thus the message that Definition 7 renders is that although soft topologies cannot be fuzzy soft topologies (they are respective subsets of different sets), they can be naturally identified with fuzzy soft topologies.

We now produce some fundamental properties that endorse the good performance of the identification of a soft topology with a fuzzy soft topology (cf., Definition 7).

Proposition 3 Suppose that $\tau_{1}, \tau_{2}$ are soft topologies on $X$. Then

1. If $\tau_{2}$ is finer than $\tau_{1}$, then $\hat{\tau}_{2}^{\eta}$ is finer than $\hat{\tau}_{1}^{\eta}$.

2. If $\mathcal{B}$ is a soft base for $\tau_{1}$ then $\eta(\mathcal{B})=\{\eta(F, E) \mid(F, E) \in$ $\mathcal{B}$ \} is a fuzzy soft base for $\hat{\tau}_{1}^{\eta}$.

Proof To prove the first claim, suppose $(f, E) \in \hat{\tau}_{1}^{\eta}$. By definition, there must exist $(F, E) \in \tau_{1}$ such that $(f, E)=\eta(F, E)$. Now $(F, E) \in \tau_{2}$ because $\tau_{2}$ is finer than $\tau_{1}$, which yields $(f, E)=\eta(F, E) \in \hat{\tau}_{2}^{\eta}$ by definition.

To prove the second claim, suppose $(f, E) \in \hat{\tau}_{1}^{\eta}$. By definition, there exists $(F, E) \in \tau_{1}$ such that $(f, E)=\eta(F, E)$. Because $\mathcal{B}$ is a soft base for $\tau_{1}$, there are $\left\{\left(F_{i}, E\right) \mid i \in I\right\} \subseteq \mathcal{B}$ such that $(F, E)=\sqcup_{i \in I}\left(F_{i}, E\right)$. Therefore $\quad(f, E)=\eta(F, E)=\eta\left(\sqcup_{i \in I}\left(F_{i}, E\right)\right)=$ $\sqcup_{i \in I}\left(\eta\left(F_{i}, E\right)\right)$ by Theorem 1 . This proves the claim that $\eta(\mathcal{B})=\{\eta(F, E) \mid(F, E) \in \mathcal{B}\}$ is a fuzzy soft base for $\hat{\tau}_{1}^{\eta}$.

Definition 2 produces the soft topology generated by a family of point-set topologies over $X$. In view of Proposition 2, now we can assure that the family produces a fuzzy soft topology over $X$ as well:

Proposition 4 Suppose that $\Sigma=\left\{\Sigma_{e}\right\}_{e \in E}$ is a family of point-set topologies on $X$. Then

$$
\begin{aligned}
& \hat{\tau}(\boldsymbol{\Sigma})=(\widetilde{\tau(\boldsymbol{\Sigma})})^{\eta}=\{\eta(F, E) \mid(F, E) \in \tau(\boldsymbol{\Sigma})\} \\
& =\left\{\eta(F, E) \mid(F, E) \in S S_{E}(X) \text { and } F(e) \in \Sigma_{e}, \forall e \in E\right\}
\end{aligned}
$$

defines a fuzzy soft topology on $X$. We call it the fuzzy soft topology on $X$ generated by $\Sigma$.

We also write $\hat{\tau}(\Sigma)=\hat{\tau}(\Sigma)$ when $\Sigma_{e}=\Sigma_{e^{\prime}}=\Sigma$ for each $e, e^{\prime} \in E$.

\subsection{The $t$-Pushes of a Soft Topological Space}

We now proceed to define a fuzzy soft topological space associated with any soft topology and $t \in[0,1]$. We shall call it the $t$-push of the soft topological space. In this way we can produce a parameterized family of fuzzy soft topologies associated with any fixed soft topology.
This construction depends upon the following auxiliary notion. Define for each $(F, E) \in S S_{E}(X)$ and $t \in[0,1]$, the fuzzy soft set $\left(F^{[t]}, E\right) \in F S_{E}(X)$ such that

$$
\begin{aligned}
F^{[t]}: & E \longrightarrow \mathcal{F}(X) \\
e & \mapsto F^{[t]}(e) \text { suchthat }^{[\mathrm{t}]}(\mathrm{e})(\mathrm{x})=\left\{\begin{array}{c}
\mathrm{t} \text { whenx } \in \mathrm{F}(\mathrm{e}), \\
0 \text { otherwise }
\end{array}\right.
\end{aligned}
$$

It is now simple to check that this assignment satisfies the following properties:

1. $\Phi^{[t]}=\hat{\Phi}$ for each $t \in[0,1]$, and $\tilde{X}^{[1]}=\hat{X}$,

2. $\left(F^{[0]}, E\right)=\hat{\Phi}$ for each $(F, E) \in S S_{E}(X)$,

3. $\left(F^{[t]}, E\right) \hat{\sqsubseteq}\left(F^{\left[t^{\prime}\right]}, E\right)$ when $t \leqslant t^{\prime}$,

4. $\left(F^{[1]}, E\right)=\eta(F, E)$ for each $(F, E) \in S S_{E}(X)$, and

5. The application of Eq. (19) to a soft point produces a fuzzy soft point when $t \in(0,1]$ : for all $x \in X$ and $t \in(0,1],\left(x^{[t]}, E\right)=\left(\{x\}_{t}, E\right)$. Notice that the case $t=$ 0 produces the null fuzzy soft set, which is not a fuzzy soft point.

The main result in this section requires some further facts concerning the concept defined by Eq. (19).

Lemma 1 Suppose $(F, E),(G, E) \in S S_{E}(X)$. Denote $(H, E)=(F, E) \sqcap(G, E) \in S S_{E}(X)$. Then for any $t \in[0,1]$, it must be the case that $\left(F^{[t]}, E\right) \hat{\sqcap}\left(G^{[t]}, E\right)=\left(H^{[t]}, E\right)$.

Proof Let us fix $e \in E$ and $x \in X$. We need to check $\left(\left(F^{[t]}, E\right) \hat{\sqcap}\left(G^{[t]}, E\right)\right)(e)(x)=\left(H^{[t]}, E\right)(e)(x)$.

First we observe that $\left(\left(F^{[t]}, E\right) \hat{\sqcap}\left(G^{[t]}, E\right)\right)(e)(x)=$ $\left.\min \left\{\left(F^{[t]}, E\right)(e)(x),\left(G^{[t]}, E\right)\right)(e)(x)\right\}$ by definition of $\hat{\Gamma}$. An inspection of (19) shows that $\left(\left(F^{[t]}, E\right) \hat{\sqcap}\left(G^{[t]}, E\right)\right)(e)(x)=t \quad$ when $\quad x \in F(e) \cap G(e)$, otherwise $\left(\left(F^{[t]}, E\right) \hat{\sqcap}\left(G^{[t]}, E\right)\right)(e)(x)=0$.

Likewise, (19) shows that $\left(H^{[t]}, E\right)(e)(x)=t$ when $x \in H(e)=F(e) \cap G(e)$, otherwise $\left(H^{[t]}, E\right)(e)(x)=0$.

Both expressions coincide thus the desired equality has been proven.

We take advantage of the technique of the proof of Lemma 1 in order to look into the performance of Eq. (19) with respect to unions:

Lemma 2 Suppose $\left(F_{i}, E\right) \in S S_{E}(X)$ for each $i \in I$. Denote $(G, E)=\sqcup_{i \in I}\left(F_{i}, E\right) \in S S_{E}(X)$. Then for any $t \in[0,1]$, it must be the case that $\cup_{i \in I}\left(F_{i}^{[t]}, E\right)=\left(G^{[t]}, E\right)$. 
Proof Let us fix $e \in E$ and $x \in X$. We need to check $\left(\hat{\cup}_{i \in I}\left(F_{i}^{[t]}, E\right)\right)(e)(x)=\left(G^{[t]}, E\right)(e)(x)$.

An inspection of (19) shows that the figure $\left(\hat{\cup}_{i \in I}\left(F_{i}^{[t]}, E\right)\right)(e)(x)$ is forcefully either $t$ or 0 . And in fact, $\left(\hat{\cup}_{i \in I}\left(F_{i}^{[t]}, E\right)\right)(e)(x)=\max \left\{F_{i}^{[t]}(e)(x) \mid i \in I\right\}$ is $t$ when $x \in F_{i}(e)$ for some $e \in E$, and 0 otherwise.

Likewise, (19) shows that $\left(G^{[t]}, E\right)(e)(x)=t$ if $x \in G(e)=\cup_{i \in I} F_{i}(e)$, and $\left(G^{[t]}, E\right)(e)(x)=0$ otherwise.

Both expressions coincide thus the desired equality has been proven.

Now we are ready to define a family of fuzzy soft topologies from any soft topology. Notice that the universe does not vary in this process.

Theorem 2 Suppose that $\tau \subseteq S S_{E}(X)$ is a soft topology. Let us fix $t \in[0,1]$. Then the family

$\hat{\tau}^{[t]}=\left\{\left(F^{[t]}, E\right) \mid(F, E) \in \tau\right\} \cup\{\hat{X}\} \subseteq F_{S S}(X)$

defines a fuzzy soft topology on $X$.

Definition 8 We say that the fuzzy soft topology $\hat{\tau}^{[t]}$ defined in Theorem 2 is the t-push associated with $\tau$.

Remark 4 Observe that because $\left(F^{[1]}, E\right)=\eta(F, E)$ for each $(F, E) \in S S_{E}(X)$ and $\tilde{X}^{[1]}=\hat{X}$, the 1-push of a soft topology $\tau$ coincides with the fuzzy soft topology associated with it (cf., Definition 7). Formally speaking: $\hat{\tau}^{[1]}=\hat{\tau}^{\eta}$ holds true for each soft topology $\tau$ on $X$.

Lemmas 1 and 2 are the key steps in the proof of Theorem 2:

Proof of Theorem 2 Let us check the three properties in Definition 5.

(1) For each $t \in[0,1], \Phi^{[t]}=\hat{\Phi} \in \hat{\tau}^{[t]}$; and $\hat{X} \in \hat{\tau}^{[t]}$ by definition.

(2) Suppose $(f, E),(g, E) \in \hat{\tau}^{[t]}$. We need to prove $(f, E) \hat{\sqcap}(g, E) \in \hat{\tau}^{[t]}$. The conclusion is evident if either $(f, E)=\hat{X}$ or $(g, E)=\hat{X}$. Let us therefore fix $(F, E),(G, E) \in \tau$ such that $(f, E)=\left(F^{[t]}, E\right)$ and $(g, E)=\left(G^{[t]}, E\right)$. Then because $\tau$ is a soft topology, the soft set $(H, E)=(F, E) \sqcap(G, E)$ satisfies $(H, E) \in \tau$. By construction, $\left(H^{[t]}, E\right) \in \hat{\tau}^{[t]}$. Now $\left(H^{[t]}, E\right)=\left(F^{[t]}, E\right) \hat{\sqcap}\left(G^{[t]}, E\right)$ by Lemma 1. In conclusion, $\left(F^{[t]}, E\right) \hat{\sqcap}\left(G^{[t]}, E\right)=(f, E) \hat{\Gamma}(g, E) \in \hat{\tau}[t]$.

(3) Suppose $\left(f_{i}, E\right) \in \hat{\tau}^{[t]}$ for each $i \in I$. We need to prove $\hat{\sqcup}_{i \in I}\left(f_{i}, E\right) \in \hat{\tau}^{[t]}$.

The conclusion is evident if $\left(f_{i}, E\right)=\hat{X}$ for some $i \in I$. Let us therefore fix $\left(F_{i}, E\right) \in \tau$ such that $\left(f_{i}, E\right)=\left(F_{i}^{[t]}, E\right)$ for each $i \in I$. Then because $\tau$ is a soft topology, the soft set $(G, E)=\sqcup_{i \in I}\left(F_{i}, E\right)$ satisfies $(G, E) \in \tau$. By construction, $\left(G^{[t]}, E\right) \in \hat{\tau}^{[t]}$. Now $\left(G^{[t]}, E\right)=\hat{\sqcup}_{i \in I}\left(F_{i}^{[t]}, E\right)$ by Lemma 2 . In conclusion, $\hat{\sqcup}_{i \in I}\left(F_{i}^{[t]}, E\right)=\hat{\sqcup}_{i \in I}\left(f_{i}, E\right) \in \hat{\tau}^{[t]}$.

It is immediate to check that if $\tau_{1}$ and $\tau_{2}$ are different soft topologies, then $\hat{\tau}_{1}^{[t]}$ and $\hat{\tau}_{2}^{[t]}$ are different for each $t \in(0,1]$. Therefore soft topologies are characterized by their $t$-pushes.

It is also easy to prove that the following fundamental relationship between soft topologies carries forward to all its $t$-pushes. The case of the 1-push has been considered before in Proposition 3:

Proposition 5 Suppose that $\tau_{1}, \tau_{2} \subseteq S S_{E}(X)$ are soft topologies on $X$. If $\tau_{2}$ is finer than $\tau_{1}$, then $\hat{\tau}_{2}^{[t]}$ is finer than $\hat{\tau}_{1}^{[t]}$ for each $t \in[0,1]$.

Proof Let us fix $t \in[0,1]$. Suppose $(f, E) \in \tau_{1}^{[t]}$. There exists $(F, E) \in \tau_{1}$ such that $(f, E)=\left(F^{[t]}, E\right)$. By the assumption that $\tau_{2}$ is finer than $\tau_{1}$ we deduce $(F, E) \in \tau_{2}$ thus $(f, E)=\left(F^{[t]}, E\right) \in \tau_{2}^{[t]}$ by construction.

\subsection{Separation Axioms: An Analysis of the $t$-Pushes Associated with a Soft Topology}

Now we demonstrate that one can infer properties of the $t$ pushes associated with a soft topology from its behavior. We do not intend to perform an exhaustive analysis, as there would be too many properties to investigate. Here we concentrate on the axioms given in Definitions 3 and 4 for illustration of this course of reasoning. They will be related to the concepts in Definition 6.

In this analysis we must be aware that except in very special cases, the $t$-pushes associated with a soft topology contradict the basic soft separation axioms in Definition 6 . To be more precise, the situation is strikingly different if we consider $t$-pushes with either $t<1$ or $t=1$.

First suppose that $\tau$ is a soft topology, $|X|>1$, and $t \in[0,1)$. Then it must be the case that $\hat{\tau}^{[t]}$ is neither $T_{0}$ nor $T_{1}$ nor $T_{2}$. It suffices to argue for $T_{0}$. When $t<1$, fix any $t^{\prime}>t$ and consider two distinct fuzzy soft points $\left(\{x\}_{t^{\prime}}, E\right)$, $\left(\{y\}_{t^{\prime}}, E\right)$ with $x \neq y$. Then it is just impossible to produce $(f, E) \in \hat{\tau}^{[t]}=\left\{\left(F^{[t]}, E\right) \mid(F, E) \in \tau\right\} \cup\{\hat{X}\}$ such that either $\left(\{x\}_{t^{\prime}}, E\right) \hat{\sqsubseteq}(f, E)$ or $\left(\{y\}_{t^{\prime}}, E\right) \hat{\sqsubseteq}(f, E)$, except $(f, E)=\hat{X}$ which does not separate one fuzzy soft point from the other.

Consider now the case of the 1-push associated with a soft topology that satisfies the axioms given in Definitions 3 and 4. Then we can prove that it satisfies the corresponding property, in the following sense: 
Proposition 6 Suppose that $\tau$ is a soft topology that satisfies $T_{0}$, resp., $T_{1}, T_{2}$. Then $\hat{\tau}^{[1]}$ satisfies $T_{0}$, resp., $T_{1}, T_{2}$.

Proof We prove the first claim. Therefore suppose that $\tau$ is a $T_{0}$ soft topology. To prove that $\hat{\tau}^{[1]}$ also satisfies $T_{0}$, let us fix $x \neq y, x, y \in X$, and $t, t^{\prime} \in(0,1]$. By assumption, either there is $(F, E) \in \tau$ with $x \in(F, E)$ and $y \notin(F, E)$, or there is $(G, E) \in \tau$ with $y \in(G, E)$ and $x \notin(G, E)$. We give the argument for the first case, the other being symmetrical.

The fact $x \in(F, E)$ implies $x \in F(e)$, hence $F^{[1]}(e)(x)=1 \geqslant x_{\{t\}}(e)(x)$, for all $e \in E$. This means that $\left(\{x\}_{t}, E\right) \hat{\check{E}}\left(F^{[1]}, E\right)$.

The fact $y \notin(F, E)$ implies $y \notin F(e)$ for some $e \in E$. Then $F^{[1]}(e)(y)=0 \geqslant y_{\left\{t^{\prime}\right\}}(e)(y)=t^{\prime}>0$ is false. This means that $\left(\{y\}_{t^{\prime}}, E\right) \hat{\sqsubseteq}\left(F^{[1]}, E\right)$ is false, which concludes the proof that $\hat{\tau}^{[1]}$ satisfies $T_{0}$.

The other two statements can be proven with routine modifications of the argument for $T_{0}$. For the purpose of proving the claim about $T_{2}$, notice that due to both Lemma $1 \quad$ and $\quad \Phi^{[1]}=\hat{\Phi}, \quad(F, E) \sqcap(G, E)=\Phi \quad$ implies $\left(F^{[1]}, E\right) \hat{\sqcap}\left(G^{[1]}, E\right)=\hat{\Phi}$.

\section{Soft Topologies Induced by Fuzzy Soft Topologies}

This section investigates soft topologies that are directly related to a given fuzzy soft topology. Our achievements are as follows. First we show that any fuzzy soft topology on $X$ induces a collection of soft topologies on $X$ indexed by $(0,1]$ that we call its $t$-throwbacks (cf., Sect. 4.1$)$. Then we prove that unfortunately, this collection does not allow us to retrieve the original fuzzy soft topology (cf., Sect. 4.3). And that it is possible to guarantee that certain properties of a fuzzy soft topology are transmitted to its $t$ throwbacks (cf., Sect. 4.4).

As in the case of Sect. 3, our fundamental construction leans on an auxiliary technical concept. This notion appeared in Feng et al. [38, Definition 4.1] under the term $t$-level soft set of a fuzzy soft set, and it allows us to associate a soft set on $X$ with each fuzzy soft set on $X$ and each membership degree $t \in[0,1]$. Hence for each $(f, E) \in$ $F S S_{E}(X)$ and $t \in[0,1]$ we define $\left(f_{[t]}, E\right) \in S S_{E}(X)$ by the expression

$$
\begin{aligned}
f_{[t]}: & E \longrightarrow \mathcal{P}(X) \\
& e \quad f_{[t]}(e)=\{x \in X \mid f(e)(x) \geqslant t\}
\end{aligned}
$$

Observe that $\hat{\Phi}_{[t]}=\Phi$ when $t \in(0,1]$. The next lemma recalls some other basic properties:

Lemma 3 Let us fix $(f, E),\left(f^{\prime}, E\right) \in F S S_{E}(X)$.
1. $\left(f_{[0]}, E\right)=\tilde{X}$.

2. For each $t, t^{\prime} \in[0,1]: t<t^{\prime}$ implies $\left(f_{\left[t^{\prime}\right]}, E\right) \sqsubseteq\left(f_{[t]}, E\right)$.

3. If $(f, E) \hat{\sqsubseteq}\left(f^{\prime}, E\right)$ then $\left(f_{[t]}, E\right) \sqsubseteq\left(f_{[t]}^{\prime}, E\right)$, for each $t \in[0,1]$.

Proof The first claim is trivial.

To prove the second claim, let us fix an arbitrary $e \in E$. Then $x \in f_{\left[t^{\prime}\right]}(e)$ is equivalent to $f(e)(x) \geqslant t^{\prime}$, which implies $f(e)(x)>t$ hence $x \in f_{[t]}(e)$. This proves $\left(f_{\left[t^{\prime}\right]}, E\right) \sqsubseteq\left(f_{[t]}, E\right)$.

To prove the third claim, let us fix $t \in[0,1]$. Then for any arbitrary $e \in E, x \in f_{[t]}(e)$ is equivalent to $f(e)(x) \geqslant t$, and because $(f, E) \hat{\sqsubseteq}\left(f^{\prime}, E\right), \quad$ we deduce $f^{\prime}(e)(x) \geqslant f(e)(x) \geqslant t$. Therefore $x \in f_{[t]}^{\prime}(e)$. This proves $\left(f_{[t]}, E\right) \sqsubseteq\left(f_{[t]}^{\prime}, E\right)$.

Example 9 below illustrates the last claim in Lemma 3.

Some further facts concerning Feng et al.'s construction in Eq. (21) will help us prove the main result in this section.

Lemma 4 Suppose $(f, E),(g, E) \in F S S_{E}(X)$. Denote $(h, E)=(f, E) \hat{\sqcap}(g, E) \in F S S_{E}(X)$. Then for any $t \in[0,1]$, it must be the case that $\left(f_{[t]}, E\right) \sqcap\left(g_{[t]}, E\right)=\left(h_{[t]}, E\right)$.

Proof Let us fix $e \in E$. We proceed by double inclusion in order to check $\left(\left(f_{[t]}, E\right) \sqcap\left(g_{[t]}, E\right)\right)(e)=\left(h_{[t]}, E\right)(e)$, i.e., $f_{[t]}(e) \cap g_{[t]}(e)=h_{[t]}(e)$.

First we fix $x \in f_{[t]}(e) \cap g_{[t]}(e)$. By (21), $f(e)(x) \geqslant t$, $g(e)(x) \geqslant t$. Since $h(e)(x)=\min \{f(e)(x), g(e)(x)\}$, it is clear that $h(e)(x) \geqslant t$ hence $x \in h_{[t]}(e)$ by (21).

The other inclusion is very similar: $x \in h_{[t]}(e)=$ $\min \{f(e)(x), g(e)(x)\}$ implies both $f(e)(x) \geqslant t$ and $g(e)(x) \geqslant t$, therefore $x \in f_{[t]}(e) \cap g_{[t]}(e)$ by (21).

The technique of the proof of Lemma 4 can be replicated so as to examine the performance of Eq. (21) with respect to unions:

Lemma 5 Suppose $\left(f^{i}, E\right) \in F S S_{E}(X)$ for each $i \in I$. Denote $(g, E)=\hat{\sqcup}_{i \in I}\left(f^{i}, E\right) \in F S S_{E}(X)$. Then for any $t \in[0,1]$, it must be the case that $\sqcup_{i \in I}\left(f_{[t]}^{i}, E\right)=\left(g_{[t]}, E\right)$.

Proof Let us fix $e \in E$. In order to check the required set equality $\sqcup_{i \in I}\left(f_{[t]}^{i}, E\right)(e)=\left(g_{[t]}, E\right)(e)$, which boils down to $\cup_{i \in I} f_{[t]}^{i}(e)=g_{[t]}(e)$, we observe the following string of equivalences.

The fact $x \in \cup_{i \in I} f_{[t]}^{i}(e)$ is equivalent to the existence of $i \in I$ such that $x \in f_{[t]}^{i}(e)$, which by (21), is equivalent to the existence of $i \in I$ such that $f^{i}(e)(x) \geqslant t$. Now this is equivalent to $\max \left\{f^{i}(e)(x): i \in I\right\} \geqslant t$, which is in turn 
equivalent to $g(e)(x) \geqslant t$ by definition of $(g, E)$. Finally, the last fact is equivalent to $x \in g_{[t]}(e)$ by (21).

In addition, for all $x \in X$ and $t^{\prime} \in(0,1]$, the application of Eq. (21) with any $t \in\left(0, t^{\prime}\right]$ to the fuzzy soft point $\left(\{x\}_{t^{\prime}}, E\right)$ produces the soft point $(x, E)$.

\subsection{Every Fuzzy Soft Topology Generates Soft Topological Spaces}

The association that Eq. (21) produces gives raise to the following construction of soft topologies from fuzzy soft topologies:

Theorem 3 Suppose that $\hat{\tau} \subseteq F S S_{E}(X)$ is a fuzzy soft topology. Let us fix $t \in(0,1]$. Then the family

$\tau_{[t]}=\left\{\left(f_{[t]}, E\right) \mid(f, E) \in \hat{\tau}\right\} \subseteq S S_{E}(X)$

defines a soft topology on $X$.

We give a name to the construction above:

Definition 9 We say that $\tau_{[t]}$ defined in Theorem 3 is the $t$-soft topology derived from the fuzzy soft topology $\hat{\tau}$. We also say that $\tau_{[t]}$ is the $t$-throwback of $\hat{\tau}$.

As it turns out, the argument of the proof of Theorem 3 uses Lemmas 4 and 5:

Proof of Theorem 3 Let us check the three properties in Definition 1.

(1) Because $t>0,\left(\hat{\Phi}_{[t]}, E\right)=\Phi$. Notice that $\Phi_{[t]}(e)=$ $\{x \in X \mid \Phi(e)(x) \geqslant t>0\}=\varnothing$ for each $e \in E$. Besides, $\left(\hat{X}_{[t]}, E\right)=\tilde{X}$.

(2) Suppose $(F, E),(G, E) \in \tau_{[t]}$. We need to prove $(F, E) \sqcap(G, E) \in \tau_{[t]}$. Let us fix $(f, E),(g, E) \in \hat{\tau}$ such that $(F, E)=\left(f_{[t]}, E\right)$ and $(G, E)=\left(g_{[t]}, E\right)$. Then because $\hat{\tau}$ is a fuzzy soft topology, the fuzzy soft set $(h, E)=(f, E) \hat{\Pi}(g, E)$ satisfies $(h, E) \in \hat{\tau}$. By construction, $\quad\left(h_{[t]}, E\right) \in \tau_{[t]}$. Now $\left(h_{[t]}, E\right)=$ $\left(f_{[t]}, E\right) \sqcap\left(g_{[t]}, E\right)$ by Lemma 4. In conclusion, $\left(f_{[t]}, E\right) \sqcap\left(g_{[t]}, E\right)=(F, E) \sqcap(G, E) \in \tau_{[t]}$.

(3) Suppose $\left(F^{i}, E\right) \in \tau_{[t]}$ for each $i \in I$. We need to prove $\sqcup_{i \in I}\left(F^{i}, E\right) \in \tau_{[t]}$. Let us fix $\left(f^{i}, E\right) \in \hat{\tau}$ such that $\left(F^{i}, E\right)=\left(f_{[t]}^{i}, E\right)$ for each $i \in I$. Then because $\hat{\tau}$ is a fuzzy soft topology, the fuzzy soft set $(h, E)=$ $\hat{\cup}_{i \in I}\left(f^{i}, E\right)$ satisfies $(h, E) \in \hat{\tau}$. By construction, $\left(h_{[t]}, E\right) \in \tau_{[t]}$. Now $\left(h_{[t]}, E\right)=\sqcup_{i \in I}\left(f_{[t]}^{i}, E\right)$ by Lemma 5. In conclusion, $\sqcup_{i \in I}\left(f_{[t]}^{i}, E\right)=\sqcup_{i \in I}\left(F^{i}, E\right) \in \tau_{[t]}$.

The construction of $t$-throwbacks has both practical and theoretical consequences. The next example shows that the $t$-throwbacks of some fuzzy soft topological spaces are soft topological spaces. Then Proposition 7 establishes that finer fuzzy soft topologies produce $t$-soft topologies that are finer.

Example 9 Suppose that $\left(f^{1}, E\right), \ldots,\left(f^{r}, E\right) \in F S S_{E}(X)$

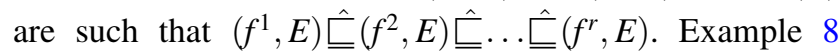
stated that $\hat{\tau}=\left\{\hat{\Phi}, \hat{X},\left(f^{1}, E\right), \ldots,\left(f^{r}, E\right)\right\}$ is a fuzzy soft topology.

Using Lemma 3, for each $t \in(0,1]$ we have

$\left(f_{[t]}^{1}, E\right) \sqsubseteq\left(f_{[t]}^{2}, E\right) \sqsubseteq \cdots \sqsubseteq\left(f_{[t]}^{r}, E\right)$.

Therefore $\tau_{[t]}$, the $t$-throwback of $\hat{\tau}$, belongs to the class of topologies defined in Example 4.

Let us compute the family of all $t$-throwbacks of $\hat{\tau}$ in the particular case where $\left(f^{1}, E\right) \sqsubseteq\left(f^{2}, E\right)$ are defined as in Table 5 (notice $X=\left\{o_{1}, o_{2}\right\}$ and $E=\left\{e_{1}, e_{2}\right\}$ ).

Consider the three soft sets defined in Table 6, also with $X=\left\{o_{1}, o_{2}\right\} \quad$ and $E=\left\{e_{1}, e_{2}\right\} . \quad$ Notice that $\left(F^{1}, E\right) \sqsubseteq\left(F^{2}, E\right) \sqsubseteq\left(F^{3}, E\right)$.

Then it is the case that:

1. When $t>0.7:\left(f_{[t]}^{i}, E\right)=\Phi, i=1,2$.

2. When $\quad t \in(0.5,0.7]: \quad\left(f_{[t]}^{1}, E\right)=\left(F^{1}, E\right)$, $\left(f_{[t]}^{2}, E\right)=\left(F^{2}, E\right)$.

3. When $t \in(0.1,0.5]:\left(f_{[t]}^{1}, E\right)=\left(f_{[t]}^{2}, E\right)=\left(F^{3}, E\right)$.

4. When $t \in(0,0.1]:\left(f_{[t]}^{1}, E\right)=\left(F^{3}, E\right),\left(f_{[t]}^{2}, E\right)=\tilde{X}$.

5. When $t=0: \quad\left(f_{[t]}^{i}, E\right)=\left(f_{[0]}^{i}, E\right)=\tilde{X}, \quad i=1,2$, by Lemma 3.

In conclusion, the $t$-throwbacks of $\hat{\tau}$ obey the following rule:

1. When either $t>0.7$ or $t=0: \tau_{[t]}=\{\Phi, \tilde{X}\}$.

2. When $t \in(0.5,0.7]: \tau_{[t]}=\left\{\Phi, \tilde{X},\left(F^{1}, E\right),\left(F^{2}, E\right)\right\}$.

3. When $t \in(0,0.5]: \tau_{[t]}=\left\{\Phi, \tilde{X},\left(F^{3}, E\right)\right\}$.

The following fundamental relationship between fuzzy soft topologies carries forward to all its $t$-throwbacks:

Proposition 7 Suppose that $\hat{\tau}^{1}, \hat{\tau}^{2} \subseteq F S_{E}(X)$ are fuzzy soft topologies on $X$. If $\hat{\tau}^{2}$ is finer than $\hat{\tau}^{1}$, then $\tau_{[t]}^{2}$ is finer than $\tau_{[t]}^{1}$ for each $t \in(0,1]$.

Proof Let us fix $t \in(0,1]$. Suppose $(F, E) \in \tau_{[t]}^{1}$. There exists $(f, E) \in \hat{\tau}^{1}$ such that $(F, E)=\left(f_{[t]}, E\right)$. By the assumption that $\hat{\tau}^{2}$ is finer than $\hat{\tau}^{1}$ we deduce $(f, E) \in \hat{\tau}^{2}$ thus $(F, E)=\left(f_{[t]}, E\right) \in \tau_{[t]}^{2}$ by construction.

\subsection{Some Relationships Involving $t$-Throwbacks and $t$-Pushes}

The constructions we have defined in Sects. 3 and 4 are related by some fundamental properties. 
First we can observe that when we start with a soft topology, then we identify it with a fuzzy soft topology (Definition 7), and afterwards we derive any $t$-soft topology from it (Definition 9), we end up with the original soft topology:

Proposition 8 Suppose that $\tau$ is a soft topology on X. Then $\left(\hat{\tau}^{\eta}\right)_{[t]}=\tau$ for each $t \in(0,1]$.

Proof The proof follows from a routine application of the concepts. Let us fix $t \in(0,1]$. Then the following string of equivalences hold true:

$(F, E) \in\left(\hat{\tau}^{\eta}\right)_{[t]}$ if and only if there exists $(f, E) \in \hat{\tau}^{\eta}$ such that $(F, E)=\left(f_{[t]}, E\right)$ if and only if there exists $\left(F^{\prime}, E\right) \in \tau$ such that $(f, E)=\eta\left(F^{\prime}, E\right)$ and $(F, E)=\left(f_{[t]}, E\right)$.

The consequence $(F, E) \in\left(\hat{\tau}^{\eta}\right)_{[t]}$ if and only if there exists $\left(F^{\prime}, E\right) \in \tau$ such that $(F, E)=\left(F^{\prime}, E\right)$ follows from the fact that $(f, E)=\eta\left(F^{\prime}, E\right)$ forcefully yields $f(e)(x) \in$ $\{0,1\}$ for each $e \in E, x \in X$, because $\left(F^{\prime}, E\right) \in S S_{E}(X)$. This means $f(e)(x) \geqslant t>0$ if and only if $f(e)(x)=1$. In other words: $x \in F(e)$ if and only if $f(e)(x)=1$ if and only if $x \in F^{\prime}(e)$, for each $e \in E, x \in X$.

Purely intuitively speaking, Proposition 8 states that any soft topology coincides with all the $t$-throwbacks that it generates, when regarded as a fuzzy soft topology. So it coincides with all the $t$-throwbacks of its 1-push.

Of course it is not generally true that all the $t$-throwbacks that a fuzzy soft topology generates coincide. We have shown this fact in Example 9.

Proposition 8 is a marked instance of a more general property whose proof is very similar. Consider the following statement:

Proposition 9 Suppose that $\tau$ is a soft topology on X. Then $\left(\hat{\tau}^{[t]}\right)_{\left[t^{\prime}\right]}=\tau$ for each $t, t^{\prime} \in(0,1]$ such that $t \geqslant t^{\prime}$.

To prove this claim along the lines of the proof of Proposition 8 , we just need to notice the following special fact:

Lemma 6 Suppose $(F, E) \in S S_{E}(X)$ and fix $t \in(0,1]$. Denote $(f, E)=\left(F^{[t]}, E\right)$. Then for any $t^{\prime} \in(0,1]$ such that $t \geqslant t^{\prime}$, it must be the case that $\left(f_{\left[t^{\prime}\right]}, E\right)=(F, E)$.

Table 5 The tabular representation of the fuzzy soft sets $\left(f^{1}, E\right),\left(f^{2}, E\right)$ in Example 9

\begin{tabular}{llllll}
\hline$\left(f^{1}, \mathrm{E}\right)$ & $e_{1}$ & $e_{2}$ & $\left(f^{2}, \mathrm{E}\right)$ & $e_{1}$ & $e_{2}$ \\
\hline$o_{1}$ & 0.7 & 0.5 & $o_{1}$ & 0.7 & 0.5 \\
$o_{2}$ & 0.5 & 0.0 & $o_{2}$ & 0.5 & 0.1 \\
\hline
\end{tabular}

Table 6 The tabular representation of the soft sets $\left(F^{1}, E\right),\left(F^{2}, E\right),\left(F^{3}, E\right)$ in Example 9

\begin{tabular}{lllllllll}
\hline$\left(F^{1}, \mathrm{E}\right)$ & $e_{1}$ & $e_{2}$ & $\left(F^{2}, \mathrm{E}\right)$ & $e_{1}$ & $e_{2}$ & $\left(F^{3}, \mathrm{E}\right)$ & $e_{1}$ & $e_{2}$ \\
\hline$o_{1}$ & 1 & 0 & $o_{1}$ & 1 & 1 & $o_{2}$ & 0 & 0 \\
$o_{2}$ & 0 & 0 & $o_{1}$ & 1 & 1 & $o_{2}$ & 1 & 0 \\
\hline
\end{tabular}

Proof Let us fix $e \in E$. In order to check the required set equality $f_{\left[t^{\prime}\right]}(e)=F(e)$, we observe that $f_{\left[t^{\prime}\right]}(e)=\left\{x \in X \mid f(e)(x) \geqslant t^{\prime}\right\}=\left\{x \in X \mid F^{[t]}(e)(x) \geqslant t^{\prime}\right\}$.

Now the facts that $F^{[t]}(e)(x)>0$ if and only if $F^{[t]}(e)(x)=t$, and $t \geqslant t^{\prime}>0$, guarantee $f_{\left[t^{\prime}\right]}(e)=F(e)$.

The application of Proposition 9 to $t=1$ produces Proposition 8.

A relationship in the other direction is ruled out because Example 10 below asssures that the following weak relationship cannot be improved:

Lemma 7 Suppose $(f, E) \in F S S_{E}(X)$ and fix $t \in(0,1]$. Denote $(F, E)=\left(f_{[t]}, E\right)$. Then for any $t^{\prime} \in(0,1]$ such that $t \geqslant t^{\prime}$, it must be the case that $\left(F^{\left[t^{\prime}\right]}, E\right) \hat{\sqsubseteq}(f, E)$.

Proof Let us fix $e \in E$. In order to check the required inequality $F^{\left[t^{\prime}\right]}(e)(x) \leqslant f(e)(x)$ for each $x \in X$, we only need to observe that

$F^{\left[t^{\prime}\right]}(e)(x)=\left\{\begin{array}{c}t^{\prime} \text { whenx } \in \mathrm{F}(\mathrm{e})=\mathrm{f}_{[\mathrm{t}]}(\mathrm{e}), \text { i.e., whenf }(\mathrm{e})(\mathrm{x}) \geqslant \mathrm{t}, \\ 0 \text { otherwise. }\end{array}\right.$

The next counterexample confirms that even if we restrict ourselves to the case $t=t^{\prime}$, the conditions of Lemma 7 do not guarantee the conclusion $\left(F^{\left[t^{\prime}\right]}, E\right)=(f, E)$ :

Example 10 Consider $\left(f^{1}, E\right) \in F S S_{E}(X)$ defined in Example 9. There we checked that its 0.6-throwback is $\left(F^{1}, E\right)$ defined in Example 9. However the 0.6-push of $\left(F^{1}, E\right)$ does not coincide with $\left(f^{1}, E\right)$.

\subsection{Non-uniqueness of the $t$-Throwbacks Associated with a Fuzzy Soft Topology}

We now provide a counterexample that shows that unfortunately, the information that the $t$-throwbacks associated with a fuzzy soft topology embody is not sufficient to retrieve such fuzzy soft topology.

To this purpose we need to show that two different fuzzy soft topologies produce exactly the same collection of $t$ throwbacks. We do this in the following example: 
Example 11 In continuation of Example 9, suppose that $\left(f^{3}, E\right) \in F S_{E}(X) \quad$ is such that $f^{3}\left(e_{1}\right)\left(x_{1}\right)=0.7=f^{3}\left(e_{2}\right)\left(x_{1}\right), \quad f^{3}\left(e_{1}\right)\left(x_{2}\right)=0.5 \quad$ and $f^{3}\left(e_{2}\right)\left(x_{2}\right)=0.2$. Therefore $\left(f^{1}, E\right) \hat{\sqsubseteq}\left(f^{3}, E\right)$, and by Example $8, \hat{\tau}^{\prime}=\left\{\hat{\Phi}, \hat{X},\left(f^{1}, E\right),\left(f^{3}, E\right)\right\}$ is a fuzzy soft topology. Notice that in fact, $\left(f^{1}, E\right) \hat{\sqsubseteq}\left(f^{2}, E\right) \hat{\sqsubseteq}\left(f^{3}, E\right)$ is true.

We can go through the computations producing the $t$ throwbacks associated with $\hat{\tau}^{\prime}$ as we did in Example 9. And we shall conclude that they coincide with the $t$-throwbacks associated with $\hat{\tau}$ defined in Example 9, for each $t \in[0,1]$. However, $\hat{\tau}$ and $\hat{\tau}^{\prime}$ are different.

\subsection{Properties of a Fuzzy Soft Topology that Transfer to its $t$-Throwbacks}

It is natural to wonder which properties of a fuzzy soft topology can be inherited by the $t$-soft topologies that it generates, i.e., by its $t$-throwbacks. The study parallels the analysis performed in Section 3.3 for property shifts in the other direction, i.e., from a soft topology to its $t$-pushes. Thus like we did in that case, for the purpose of illustration of the technique we concentrate on the implications of the fundamental separation axioms (cf., Definition 6).

Proposition 10 Suppose that $\hat{\tau}$ is a fuzzy soft topology that satisfies $T_{0}$, resp., $T_{1}, T_{2}$. Then for any $t \in(0,1]$, the soft topology $\tau_{[t]}$ satisfies $T_{0}$, resp., $T_{1}, T_{2}$.

Proof We prove the first claim. Therefore suppose that $\hat{\tau}$ is a $T_{0}$ fuzzy soft topology and fix $t \in(0,1]$. To prove that $\tau_{[t]}$ also satisfies $T_{0}$, let us fix $x \neq y, x, y \in X$. Consider the distinct fuzzy soft points $\left(\{x\}_{t}, E\right)$ and $\left(\{y\}_{t}, E\right)$.

By assumption, either there is $(f, E) \in \hat{\tau}$ with $\left(\{x\}_{t}, E\right) \hat{\sqsubseteq}(f, E)$ such that $\left(\{y\}_{t}, E\right) \hat{\sqsubseteq}(f, E)$ is false, or there is $(g, E) \in \hat{\tau}$ with $\left(\{y\}_{t}, E\right) \hat{\complement}(g, E)$ such that $\left(\{x\}_{t}, E\right) \hat{\complement}(g, E)$ is false. We give the argument for the first case, the other being symmetrical.

The fact that $(f, E) \in \hat{\tau}$ satisfies $\left(\{x\}_{t}, E\right) \hat{\sqsubseteq}(f, E)$ is equivalent to $f(e)(x) \geqslant t$, for all $e \in E$. This means that $x \in f_{[t]}(e)$ for all $e \in E$ by Eq. (21), hence $x \in\left(f_{[t]}, E\right)$.

The fact that $(f, E) \in \hat{\tau}$ does not satisfy $\left(\{y\}_{t}, E\right) \hat{\complement}(f, E)$ is equivalent to the existence of $e \in E$ such that $f(e)(y)<t$. This means that $y \in f_{[t]}(e)$ for all $e \in E$ is false, hence $y \notin\left(f_{[t]}, E\right)$, which concludes the proof that $\tau_{[t]}$ satisfies $T_{0}$.

The other statements can be proven with routine modifications of the argument for $T_{0}$. For the purpose of proving the claim about $T_{2}$, notice that due to Lemma 3 and $\hat{\Phi}_{[t]}=\Phi$ because $t \in(0,1]$, the fact that $(f, E) \hat{\sqcap}(g, E)=\hat{\Phi}$ implies $\left(f_{[t]}, E\right) \sqcap\left(g_{[t]}, E\right)=\Phi$.

\section{Conclusion}

To the best of our knowledge this paper conducts the first systematic analysis of the relationships between soft topologies and fuzzy soft topologies. We have shown that this long-forgotten problem produces a rich variety of fundamental results thus it deserves further investigation.

Let us summarize our main findings. Consider first a soft topology $\tau$.

1. We only need to resort to a 'folk' theorem to prove that $\tau$ may be identified with a fuzzy soft topology.

2. More generally: $\tau$ induces a family of fuzzy soft topologies, one for each $t \in[0,1]$, called its $t$-pushes.

(2.1) The 1-push of $\tau$ is the fuzzy soft topology associated with it.

(2.2) When $t \in[0,1)$ and $X$ has more than one element, the $t$-push of $\tau$ is neither $T_{0}$ nor $T_{1}$ nor $T_{2}$.

(2.3) However the 1-push of $\tau$ is $T_{0}$, resp., $T_{1}, T_{2}$, when $\tau$ is $T_{0}$, resp., $T_{1}, T_{2}$. This construction satisfies the following properties:

(2.4) Different soft topologies produce different $t$ pushes.

(2.5) Finer soft topologies produce finer $t$-pushes (for any fixed $t \in[0,1]$ ).

Consider now a fuzzy soft topology $\hat{\tau}$.

3. It induces a family of soft topologies, one for each $t \in(0,1]$, called its $t$-throwbacks.

(3.1) The $t$-throwbacks of $\hat{\tau}$ are $T_{0}$, resp., $T_{1}, T_{2}$, when $\hat{\tau}$ is $T_{0}$, resp., $T_{1}, T_{2}$. This construction satisfies the following properties:

(3.2) Different fuzzy soft topologies may produce the same $t$-throwbacks.

(3.3) Finer fuzzy soft topologies produce finer $t$ throwbacks (for any fixed $t \in[0,1]$ ).

In addition to these facts, some relationships among these two constructions hold true:

4. Any soft topology coincides with all the $t$-throwbacks of its 1-push. This is a particular statement of a more general property: any soft topology coincides with all the $t^{\prime}$-throwbacks of any fixed $t$-push of it, when $t \geqslant t^{\prime}>0$.

Further investigation can be done concerning various aspects of our analysis. The studies carried on in Sects. 3.3 and 4.4 can be extended to comprise further axioms. The disappointing fact that the $t$-throwbacks of a fuzzy soft topology do not characterize it launches the question of whether other soft topologies allowing us to recover the original fuzzy soft topology can be designed. Besides, our 
study can inspire comparable analysis concerning for example bipolar fuzzy soft topologies, hesitant fuzzy soft topologies, or $N$-soft topologies. We expect to return to these issues in the future.

\section{Notation and Conventions}

$\sqcap, \sqcup, \sqsubseteq$ denote the soft intersection, union, and inclusion.

$\hat{\Pi}, \hat{\cup}, \hat{\complement}$ denote the fuzzy soft intersection, union, and inclusion.

$(F, E),(G, E),(H, E),\left(F_{i}, E\right),\left(F^{i}, E\right), \ldots$ denote soft sets. The first component (mapping) is capitalized. Also, $(x, E)$ denotes the soft point with $x(e)=\{x\}$ for all $e \in E$.

$(f, E),\left(f_{i}, E\right),\left(f^{i}, E\right),\left(f^{\prime}, E\right), \ldots$ denote fuzzy soft sets. The first component (mapping) is not capitalized. Also, $\left(\{x\}_{t}, E\right)$ denotes the fuzzy soft point with $\{x\}_{t}(e)(x)=t$ for all $e \in E,\{x\}_{t}(e)\left(x^{\prime}\right)=0$ otherwise.

$(x, E),(y, E), \ldots$ denote soft points.

$\left(\{x\}_{t}, E\right),\left(\{y\}_{t^{\prime}}, E\right), \ldots$ denote fuzzy soft points: cf., Eq. (7).

$\Phi, \tilde{X} \in \tau$ denote the null and absolute soft sets, respectively.

$\hat{\Phi}, \hat{X} \in \tau$ denote the null and absolute fuzzy soft sets, respectively.

$\Sigma, \Sigma^{\prime}, \Sigma_{e}, \ldots$. denote crisp topologies.

$\tau, \tau^{\prime}, \tau^{i}, \ldots$. denote soft topologies.

$\hat{\tau}, \hat{\tau}^{\prime}, \hat{\tau}^{i}, \ldots$. denote fuzzy soft topologies. We distinguish them from soft topologies by putting a hat on them.

$\hat{\tau}^{\eta}$ is the fuzzy soft topology associated with $\tau$ (cf., Definition 7).

$\hat{\tau}^{[t]}$ is the $t$-push associated with the soft topology $\tau$ (cf., Theorem 2). It is a fuzzy soft topology.

$\tau_{[t]}$ is the $t$-throwback of the fuzzy soft topology $\hat{\tau}$ (cf., Theorem 3). It is a soft topology.

Funding Open Access funding provided thanks to the CRUE-CSIC agreement with Springer Nature. The author is grateful to the Junta de Castilla y León and the European Regional Development Fund (Grant CLU-2019-03) for the financial support to the Research Unit of Excellence "Economic Management for Sustainability" (GECOS).

Data Availability Not applicable.

Code Availability Not applicable.

\section{Declarations}

Conflict of interest The author declares no conflict of interest.

Open Access This article is licensed under a Creative Commons Attribution 4.0 International License, which permits use, sharing, adaptation, distribution and reproduction in any medium or format, as long as you give appropriate credit to the original author(s) and the source, provide a link to the Creative Commons licence, and indicate if changes were made. The images or other third party material in this article are included in the article's Creative Commons licence, unless indicated otherwise in a credit line to the material. If material is not included in the article's Creative Commons licence and your intended use is not permitted by statutory regulation or exceeds the permitted use, you will need to obtain permission directly from the copyright holder. To view a copy of this licence, visit http://creativecommons. org/licenses/by/4.0/.

\section{References}

1. Hausdorff, F.: Grundzüge der mengenlehre. Veit, Leipzig (1914)

2. Bergstrom, T.C.: Maximal elements of acyclic relations on compact sets. J. Econ. Theory 10, 403-404 (1975)

3. Walker, M.: On the existence of maximal elements. J. Econ. Theory 16(2), 470-474 (1977)

4. Alcantud, J.C.R.: Characterization of the existence of maximal elements of acyclic relations. Econ. Theory 19(2), 407-416 (2002). https://doi.org/10.1007/PL00004219

5. Zadeh, L.A.: Fuzzy sets. Inf. Control 8, 338-353 (1965). https:// doi.org/10.1016/S0019-9958(65)90241-X

6. Chang, C.L.: Fuzzy topological spaces. J. Math. Anal. Appl. 24(1), 182-s190 (1968)

7. Goguen, J.A.: Fuzzy Tychonoff theorem. J. Math. Anal. Appl. 43(3), 734-742 (1973). https://doi.org/10.1016/0022247X(73)90288-6

8. Lowen, R.: Fuzzy topological spaces and fuzzy compactness. J. Math. Anal. Appl. 56(3), 621-633 (1976). https://doi.org/10. 1016/0022-247X(76)90029-9

9. Çoker, D.: An introduction to intuitionistic fuzzy topological spaces. Fuzzy Sets Syst. 88(1), 81-89 (2011). https://doi.org/10. 1016/S0165-0114(96)00076-0

10. Shabir, M., Naz, M.: On soft topological spaces. Comput. Math. Appl. 61(7), 1786-1799 (2011). https://doi.org/10.1016/j.camwa. 2011.02.006

11. Molodtsov, D.: Soft set theory-first results. Comput. Math. Appl. 37, 19-31 (1999)

12. Das, S., Samanta, S.: Soft metric. Ann. Fuzzy Math. Inf. 6, 77-94 (2013)

13. Nazmul, S., Samanta, S.: Some properties of soft topologies and group soft topologies. Ann. Fuzzy Math. Inf. 8, 645-661 (2014)

14. Zorlutuna, I., Akdag, M., Min, W.K., Atmaca, S.: Remarks on soft topological spaces. Ann. Fuzzy Math. Inf. 3, 171-185 (2012)

15. Aygünoğlu, A., Aygün, H.: Some notes on soft topological spaces. Neural Comput. Appl. 21(1), 113-119 (2012). https://doi. org/10.1007/s00521-011-0722-3

16. Nazmul, S., Samanta, S.: Neighbourhood properties of soft topological spaces. Ann. Fuzzy Math. Inf. 6, 1-15 (2013)

17. Al-shami, T.M., El-Shafei, M.E.: Partial belong relation on soft separation axioms and decision-making problem, two birds with one stone. Soft Comput. 24, 5377-5387 (2020). https://doi.org/ 10.1007/s00500-019-04295-7

18. Hussain, S., Ahmad, B.: Some properties of soft topological spaces. Comput. Math. Appl. 62, 4058-4067 (2011)

19. Terepeta, M.: On separating axioms and similarity of soft topological spaces. Soft Comput. 23(3), 1049-1057 (2019). https:// doi.org/10.1007/s00500-017-2824-z

20. Al-shami, T.M., El-Shafei, M., Abo-Elhamayel, M.: Seven generalized types of soft semi-compact spaces. Korean J. Math. 27(3), 661-690 (2019)

21. Alcantud, J.C.R.: Soft open bases and a novel construction of soft topologies from bases for topologies. Mathematics 8(5), 672 (2020) 
22. Kočinac, L.D.R., Al-shami, T.M., Çetkin, V.: Selection principles in the context of soft sets: Menger spaces. Soft Comput. (2021). https://doi.org/10.1007/s00500-021-06069-6

23. Tanay, B., Kandemir, M.B.: Topological structure of fuzzy soft sets. Comput. Math. Appl. 61(10), 2952-2957 (2011). https://doi. org/10.1016/j.camwa.2011.03.056

24. Khameneh, A.Z., Kiliçman, A., Salleh, A.R.: An adjustable approach to multi-criteria group decision-making based on a preference relationship under fuzzy soft information. Int. J. Fuzzy Syst. 19(6), 1840-1865 (2017). https://doi.org/10.1007/s40815016-0280-z

25. Hassan, N., Sayed, O.R., Khalil, A.M., Ghany, M.A.: Fuzzy soft expert system in prediction of coronary artery disease. Int. J. Fuzzy Syst. 19(5), 1546-1559 (2017). https://doi.org/10.1007/ s40815-016-0255-0

26. Khameneh, A.Z., Kiliçman, A., Salleh, A.R.: Fuzzy soft product topology. Ann. Fuzzy Math. Inf. 7(6), 935-947 (2014)

27. Roy, S., Samanta, T.K.: A note on fuzzy soft topological spaces. Ann. Fuzzy Math. Inf. 3(2), 305-311 (2012)

28. Riaz, M., Tehrim, S.T.: On bipolar fuzzy soft topology with decision-making. Soft Comput. 24, 18259-18272 (2020). https:// doi.org/10.1007/s00500-020-05342-4

29. Riaz, M., Davvaz, B., Fakhar, A., Firdous, A.: Hesitant fuzzy soft topology and its applications to multi-attribute group decisionmaking. Soft Comput. 24, 16269-16289 (2020). https://doi.org/ 10.1007/s00500-020-04938-0

30. Riaz, M., Çağman, N., Zareef, I., Aslam, M.: $N$-soft topology and its applications to multi-criteria group decision making. J. Intell. Fuzzy Syst. 36, 6521-6536 (2019). https://doi.org/10.3233/JIFS182919

31. Fatimah, F., Rosadi, D., Hakim, R.B.F., Alcantud, J.C.R.: $N$-soft sets and their decision making algorithms. Soft Comput. 22, 3829-3842 (2018). https://doi.org/10.1007/s00500-017-2838-6

32. Munkres, J.: Topology. Featured titles for topology. Prentice Hall, Hoboken (2000)

33. Willard, S.: General topology. Addison-Wesley series in mathematics. Dover Publications, Mineola (2004)

34. Maji, P., Biswas, R., Roy, A.: Soft set theory. Comput. Math. Appl. 45, 555-562 (2003)
35. Çağman, N., Karataş, S., Enginoglu, S.: Soft topology. Comput. Math. Appl. 62(1), 351-358 (2011). https://doi.org/10.1016/j. camwa.2011.05.016

36. Roy, S., Samanta, T.K.: A note on a soft topological space. Punjab Univ. J. Math. 46(1), 19-24 (2014)

37. Maji, P., Biswas, R., Roy, A.R.: Fuzzy soft sets. J. Fuzzy Math. 9, 589-602 (2001)

38. Feng, F., Jun, Y.B., Liu, X., Li, L.: An adjustable approach to fuzzy soft set based decision making. J. Comput. Appl. Math. 234(1), 10-20 (2010). https://doi.org/10.1016/j.cam.2009.11.055

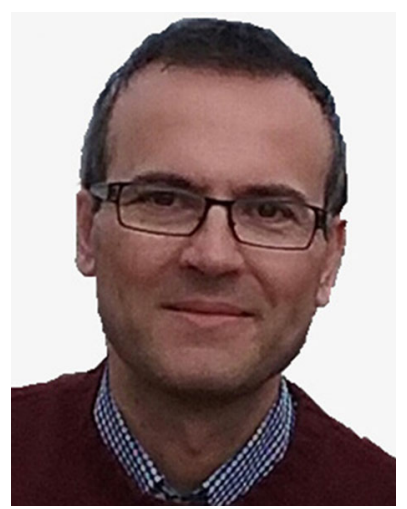

José Carlos R. Alcantud received his M.Sc. in Mathematics in 1991 from the University of Valencia, Spain and Ph.D. in Mathematics in 1996 from the University of Santiago de Compostela, Spain. He is currently a professor in the Department of Economics and Economic History, with the University of Salamanca, Spain. $\mathrm{He}$ has authored over 110 papers on topics including mathematical economics, social choice, fuzzy mathematics and soft computing. He serves on the editorial board of Symmetry, Foundations, Journal of Computational and Cognitive Engineering, and Global \& Local Economic Review, and on the advisory board of Sci. He has been a reviewer for more than 60 SCI journals, including Artificial Intelligence Review, Fuzzy Sets and Systems, IEEE Transactions on Fuzzy Systems, Information Fusion, Information Sciences, Knowledge-Based Systems, etc. As the project leader, he has directed seven research projects at the national or provincial level. He has also been a member of a EU-funded reseach project. 